INRA Prod. Anim., 2011, 24 (5), 461-474

\title{
Leviers d'action pour réduire la production de méthane entérique par les ruminants
}

\author{
M. DOREAU, C. MARTIN, M. EUGÈNE, M. POPOVA, D.P. MORGAVI \\ INRA, UR1213, Herbivores, F-63122 Saint-Genès-Champanelle, France \\ Courriel : michel.doreau@clermont.inra.fr
}

L'un des moyens de réduire les émissions de gaz à effet de serre par les ruminants est de diminuer les émissions de méthane entérique. De nombreuses solutions sont préconisées, au niveau de la ration ou du type d'animal, mais la réponse de la production de méthane dans le rumen est variable. Il n'y a pas de solution miracle, mais les principales pistes sont présentées.

La part de l'élevage dans les émissions de Gaz à Effet de Serre (GES) responsables du réchauffement climatique fait toujours débat, car elle dépend du mode de calcul adopté et de la zone géographique considérée. Mais quelle que soit cette part, elle est suffisamment importante pour qu'il soit nécessaire de rechercher les moyens de réduire ces émissions; nombreuses sont les préconisations faites par des organismes officiels internationaux comme la FAO (Food and Agriculture Organization), le GIEC (Groupe d'experts Intergouvernemental sur l'Evolution du Climat, en anglais IPCC), ou l'UNFCCC (Commission des Nations Unies sur le changement climatique). Parallèlement, de nombreuses nations se sont engagées à une réduction des émissions ; les directives de l'Union Européenne se sont traduites dans les différents pays membres par des actions incitatives fondées ou non sur des aides, ou de simples feuilles de route avec des objectifs chiffrés impliquant les différents acteurs des filières (Doreau et Dollé 2011). Dans le cas des ruminants, lorsque la totalité des émissions de GES est exprimée en équivalent gaz carbonique sur la base du pouvoir réchauffant respectif du gaz carbonique, du protoxyde d'azote et du méthane, ce dernier représente environ la moitié des émissions. Il est donc justifié de rechercher des leviers d'action pour les réduire. C'est l'objet de cet article consacré aux émissions de méthane entérique par les ruminants ; ces derniers contribuent à plus de $97 \%$ des émissions de méthane des animaux d'élevage en France selon Vermorel et al (2008). Les propositions pour agir sur le méthane entérique sont toutefois à replacer dans le cadre plus global de la réduction de l'ensemble des GES, qui est analysé dans l'article de Dollé et al (2011).
Depuis plusieurs années, de nombreux leviers d'action sont testés dans le monde entier pour réduire les émissions de méthane entérique, avec en particulier une recherche abondante sur les additifs alimentaires. Les essais in vitro, très utiles pour explorer en conditions contrôlées les effets d'un nombre important de substances, mettent souvent en évidence des effets significatifs sur la production de méthane, mais les conclusions tirées sont parfois hâtives. Les essais in vivo sont nombreux avec des rations classiques, beaucoup plus limités dans le cas des additifs; les essais à long terme sont très rares. Cet article fait le point de l'état des connaissances sur l'efficacité de nombreux leviers testés pour réduire les émissions de méthane, et dégagera des pistes pour l'avenir. Nous nous centrerons sur les résultats obtenus in vivo, car de nombreuses techniques permettant de réduire le méthane in vitro ne se révèlent pas efficaces in vivo ; en effet les systèmes in vitro les plus utilisés reproduisent imparfaitement les processus physiologiques dans le rumen. De très nombreux travaux sont disponibles; seuls sont cités les plus représentatifs ou les plus originaux. Pour une bibliographie plus exhaustive, le lecteur se reportera aux revues de Beauchemin et al (2009), de Martin et al (2010) et de Cottle et al (2011).

La réduction de la production de méthane peut être mesurée par $\mathrm{kg}$ de Matière Sèche (MS) ingérée, ce qui permet d'évaluer l'impact d'une pratique sur les processus digestifs, ou par $\mathrm{kg}$ de lait ou de viande produits, ce qui intègre l'efficacité de production. Par ailleurs, les stratégies de réduction des émissions de méthane par l'alimentation peuvent dans certains cas être effi- caces uniquement à court terme, en raison des possibilités d'adaptation de l'écosystème microbien du rumen (cf. article de Popova et al 2011). La réduction ciblée d'une communauté microbienne entraîne souvent une modification de l'équilibre entre les autres communautés, ou bien n'est que transitoire, et donc la réduction de la production de méthane n'est pas permanente. Les mesures de production de méthane sont réalisées le plus souvent après 2 à 4 semaines d'application du traitement étudié. On précisera donc si les effets obtenus ont été confirmés sur le long terme, c'est-à-dire après plusieurs mois d'application du traitement. Enfin, l'acceptabilité par le consommateur des techniques de réduction de la production de méthane sera évoquée; certaines stratégies efficaces font en effet appel à des additifs non autorisés, ou peuvent présenter un risque d'effets délétères sur la santé de l'animal ou du consommateur.

\section{1 / Stratégies de réduction de la production de métha- ne}

Le méthane est produit dans le rumen par les Archaea méthanogènes à partir de l'hydrogène issu de la fermentation des glucides. La voie biochimique de l'acétate produit de l'hydrogène, alors que celle du propionate en consomme, ce qui explique la relation positive entre la production de méthane et le rapport acétate/propionate du rumen (Sauvant et al 2011). Les effets des différentes stratégies à l'étude pour agir sur la structure ou l'activité de la communauté méthanogène ont été détaillés par Popova et al (2011). L'hydrogène étant 
Figure 1. Relation entre la population de protozoaires et la production de méthane dans le rumen : bilan de la bibliographie disponible (d'après Morgavi et al 2010a).

Les différents essais sont identifiés par des chiffres à l'intérieur de symboles selon le facteur de variation étudié : triangles pour les concentrés riches en amidon, ronds pour les lipides, carrés pour les extraits de plantes. La ligne pointillée représente la droite de régression après prise en compte de l'effet essai.

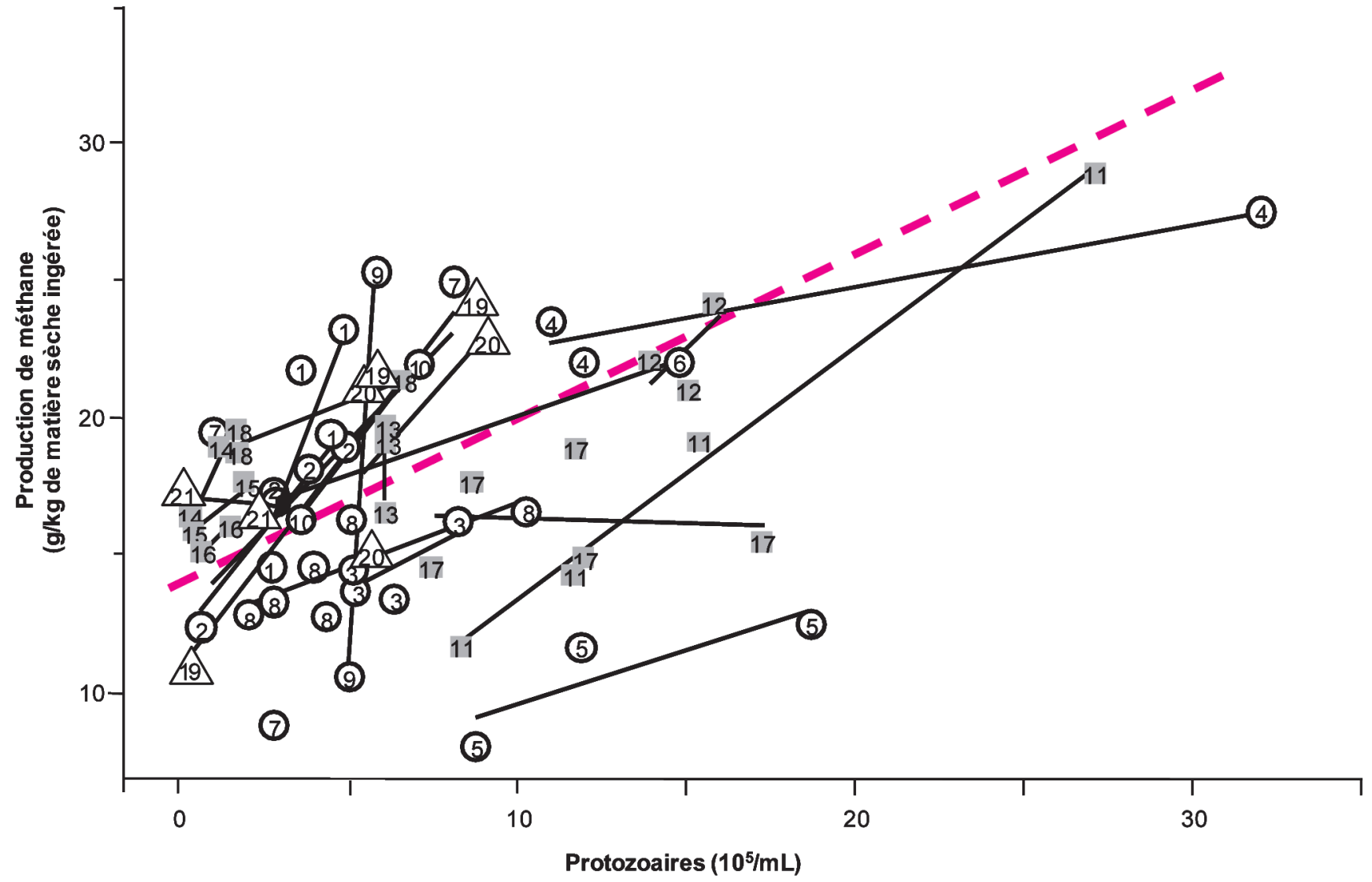

un substrat limitant dans la voie métabolique de la méthanogenèse, deux modes d'action majeurs seront discutés ci-dessous : i) réduire la production nette d'hydrogène ou $\mathrm{ii}$ ) réorienter l'hydrogène vers d'autres voies métaboliques potentiellement bénéfiques pour l'animal.

La production nette d'hydrogène peut être réduite suite à une suppression partielle ou totale de la population de protozoaires (défaunation) dans le rumen. Une analyse de la bibliographie montre un lien étroit entre la taille de la population de protozoaires et l'émission de méthane (figure 1). En effet, les protozoaires sont de forts producteurs d'hydrogène, en partie parce qu'ils favorisent le butyrate dans le mélange d'Acides Gras Volatils (AGV). La suppression des protozoaires par des techniques de défaunation totale utilisées en expérimentation n'est pas réalisable dans la pratique (Popova et al 2011). Mais dans des conditions d'alimentation applicables sur le terrain, la population de protozoaires peut être réduite avec des rations très riches en céréales, ou par l'effet toxique de certains composés : Acides Gras (AG) à chaîne moyenne (laurique et myristique) ou polyinsaturés à 18 carbones (linoléique et linolénique), ou avec des composés secondaires des plantes comme les saponines. La défaunation partielle ou totale a deux principales conséquences sur l'équilibre de l'écosystème microbien ruminal. D'une part la population bactérienne se trouve accrue, en raison de l'absence de prédation par les protozoaires ; d'autre part du fait de cette amélioration de la synthèse de biomasse microbienne, qui joue un rôle de «puits d'hydrogène», il y a moins d'hydrogène disponible pour la méthanogenèse. Les profils des AGV sont plus pauvres en butyrate, au profit de l'acétate ou du propionate (Jouany 1994). Malgré les diminutions observées dans la production de méthane, la réduction de la population de protozoaires a, en outre, deux conséquences générales qu'il est nécessaire de garder en mémoire: une réduction de la digestibilité des parois, et une augmentation du flux de protéines quittant le rumen, car les protozoaires sont des prédateurs des bactéries, et sortent lentement du rumen.

En ce qui concerne la réorientation de l'hydrogène vers d'autres voies métabo- liques, l'augmentation de la part du propionate dans le profil des AGV est une première solution. Le lien entre nature de l'alimentation ou additifs alimentaires et proportion de propionate est établi depuis longtemps. Le tableau 1 fait le point des principaux facteurs connus. Cette orientation vers le propionate est souvent liée à une diminution du $\mathrm{pH}$ (cas des rations riches en concentré). Le $\mathrm{pH}$ a par ailleurs un effet spécifique : des $\mathrm{pH}$ faibles entraînent une diminution des protozoaires et des méthanogènes (Van Kessel et Russell 1996).

Un autre moyen pour réorienter l'hydrogène disponible pour la production de méthane est de l'utiliser dans d'autres voies biochimiques, comme la voie de l'acétogenèse ou les voies réductrices de sulfates ou de nitrates (Morgavi et al 2010a). Les limites et les avantages de ces stratégies sont discutés dans l'article de Popova et al (2011). Il est à noter que l'hydrogénation des AG polyinsaturés ne compte que marginalement dans la captation d'hydrogène «puits à hydrogène» permettant de limiter la méthanogenèse: dans une ration de vaches laitières riche en $A G$ polyinsaturés, la captation de l'hydrogène 
Tableau 1. Moyens d'accroître la proportion de propionate dans les AGV produits dans le rumen.

\begin{tabular}{|l|}
\hline Augmentation du pourcentage de concentré \\
\hline Parmi les fourrages, forte digestibilité (herbe jeune) \\
\hline Parmi les concentrés glucidiques, richesse en amidon (céréales) \\
\hline Parmi les céréales, vitesse de digestion dans le rumen élevée (blé, orge) \\
\hline Addition d'acides gras polyinsaturés à 18 carbones (tournesol, lin) \\
\hline Antibiotiques ionophores \\
\hline Acides organiques \\
\hline
\end{tabular}

nécessaire pour saturer ces $\mathrm{AG}$ ne réduirait la production de méthane que de 5 à $6 \%$.

\section{2 / Composition de la ration}

\section{1 / Proportion de concentré et nature des glucides}

L'accroissement de la proportion de concentré dans la ration entraîne une diminution de la production de méthane par $\mathrm{kg}$ de MS ingérée, et de manière encore plus nette en $\%$ de l'énergie brute ingérée. Une relation curvilinéaire a été mise en évidence par Sauvant et Giger-Reverdin (2007) : la méthanogenèse est fortement réduite pour des niveaux de concentré très élevés. Ceci est pris en compte dans la méthode d'estimation du GIEC dite «Tier 2» qui propose d'estimer les émissions de méthane à $5 \%$ de l'énergie brute ingérée pour l'ensemble des rations, excepté pour les rations de type «feedlot» très riches en concentré, pour lesquelles elles sont estimées à $3 \%$ de l'énergie brute ingérée (IPCC, 2006). Ces chiffres correspondent d'ailleurs aux résultats obtenus par Doreau et al (2011b) sur taurillons à l'engrais: de l'ordre de $6 \%$ pour des rations à base d'ensilage de maïs ou de foin, et $3 \%$ pour une ration à $86 \%$ de concentré. Une variation similaire, quoique moins nette, a été observée par Lovett et al (2003) avec des rapports entre les proportions de fourrage et de concentré dans la ration allant de 65/35 à 10/90. Dans ce cas, la diminution de la méthanogenèse est particulièrement marquée pour les forts niveaux d'ingestion, comme cela a été mis en évidence par Sauvant et Giger-Reverdin (2009). Cette interaction entre les effets de la digestibilité de la ration, qui augmente avec la proportion de concentré, et du niveau alimentaire avait été montrée pour la première fois par Blaxter et al (1965). Selon la méta-analyse de Sauvant et Giger-Reverdin (2009), pour un niveau alimentaire inférieur ou égal à $2,5^{1}$, il y a peu de variation de la production de méthane en fonction de l'énergie brute ingérée (de 6 à 7\%) pour des niveaux de concentré compris entre 20 et $50 \%$ de la ration, ce qui correspond à la réalité du terrain en France pour les vaches laitières. Ces différents résultats sont liés à l'effet du pourcentage de concentré sur le rapport acétate/propionate dans les $\mathrm{AGV}$ du rumen, et à la diminution du $\mathrm{pH}$ à des niveaux de concentré dans la ration très élevés.

De nombreuses études anciennes ont montré que les céréales sont à l'origine d'émissions de méthane plus faibles par $\mathrm{kg}$ de glucides digestibles que les concentrés riches en parois (Moe et Tyrrell 1979). Toutefois il y a eu peu de comparaisons directes. Beever et al (1989) ont montré que le remplacement de pulpes de betteraves par de l'orge pour une ration riche en concentré réduisait l'émission de méthane par $\mathrm{kg}$ de MS de 34\%. L'orientation fermentaire vers l'acétate dans le cas de la pulpe, ou vers le propionate dans le cas de l'orge en est certainement la cause. Beauchemin et McGinn (2005) ont montré que la production de méthane par kg de MS ou en \% de l'énergie brute ingérée était plus faible avec du maïs qu'avec de l'orge pour des génisses engraissées avec des rations très riches en concentré. Les céréales ayant été distribuées aplaties, ce résultat est probablement dû au fait qu'une partie du maïs était digéré dans l'intestin. Avec des rations à $50 \%$ de concentré, Moe et al (1973) n'ont pas observé de différence entre du maïs et de l'avoine, mais les deux aliments étaient broyés, ce qui entraîne une forte dégradation ruminale dans les deux cas. Enfin, Johnson et
Johnson (1995) suggèrent que les sucres solubles sont plus méthanogènes que l'amidon. Bien qu'il manque les données pour l'affirmer, ceci pourrait être dû à des différences dans les orientations des fermentations ruminales, vers le butyrate pour les sucres solubles et vers le propionate pour l'amidon. En conclusion, les différences selon la nature des glucides du concentré existent, mais elles sont plus modestes que les différences liées à la proportion de concentré dans la ration.

\section{2 / Nature du fourrage}

Peu d'essais ont comparé l'effet de différents types de fourrages sur les émissions de méthane d'animaux en production. Moe et Tyrrell (1979) concluaient à partir d'une base de données personnelle que des rations à base de foin ou d'ensilage de maïs avaient des effets similaires sur la production de méthane ; mais Martin et al (2009) ont observé une production de méthane par $\mathrm{kg}$ de lait plus élevée pour des vaches recevant une ration à base de foin que pour d'autres vaches recevant une ration à base d'ensilage de maïs, et Doreau et al (non publié) ont montré que la production de méthane par $\mathrm{kg}$ de lait était plus élevée pour une ration à base d'ensilage d'herbe que pour une ration à base d'ensilage de maïs (figure 2). Ces résultats pourraient s'expliquer par la fraction amylacée des ensilages de céréales, qui orientent les fermentations ruminales vers le propionate. Inversement, Chung et al (2011a) ont observé avec des vaches taries une production de méthane par $\mathrm{kg}$ de $\mathrm{MS}$ plus élevée de $33 \%$ avec de l'ensilage d'orge qu'avec du foin ; dans cet essai les proportions des AGV étaient similaires entre les deux régimes. Enfin, Staerfl et al (2012) ont observé avec des taurillons au cours de l'engraissement des différences très modérées de production de méthane par kg de MS entre des rations à base d'ensilage d'herbe ou d'ensilage de maïs. En conclusion, des recherches complémentaires sur l'effet de la ration de base restent à mener ; dans l'état actuel des connaissances, il ne semble pas que la nature du fourrage de la ration soit un déterminant majeur de l'émission de méthane.

La composition chimique du fourrage, liée à son stade de maturité, influence modérément les émissions de méthane. Une récente méta-analyse réalisée sur des fourrages distribués sans complémentation a montré que la production de méthane par kg de matière organique ingérée ou digérée était corrélée positivement à la digestibilité de la

\footnotetext{
${ }^{1}$ Par définition, un niveau alimentaire de 1 correspond aux quantités ingérées nécessaires pour couvrir les besoins d'entretien de l'animal.
} 
Figure 2. Effet de la nature du fourrage sur la production de méthane.

Comparaisons 1 à 4 : essai 1 (Martin et al 2009 et non publié); comparaisons 5 et 6 : essai 2 (Doreau et al non publié). Les comparaisons 2, 3 et 4 sont des rations supplémentées en lipides. Tous les régimes sont formulés pour couvrir les besoins énergétiques et azotés des animaux.

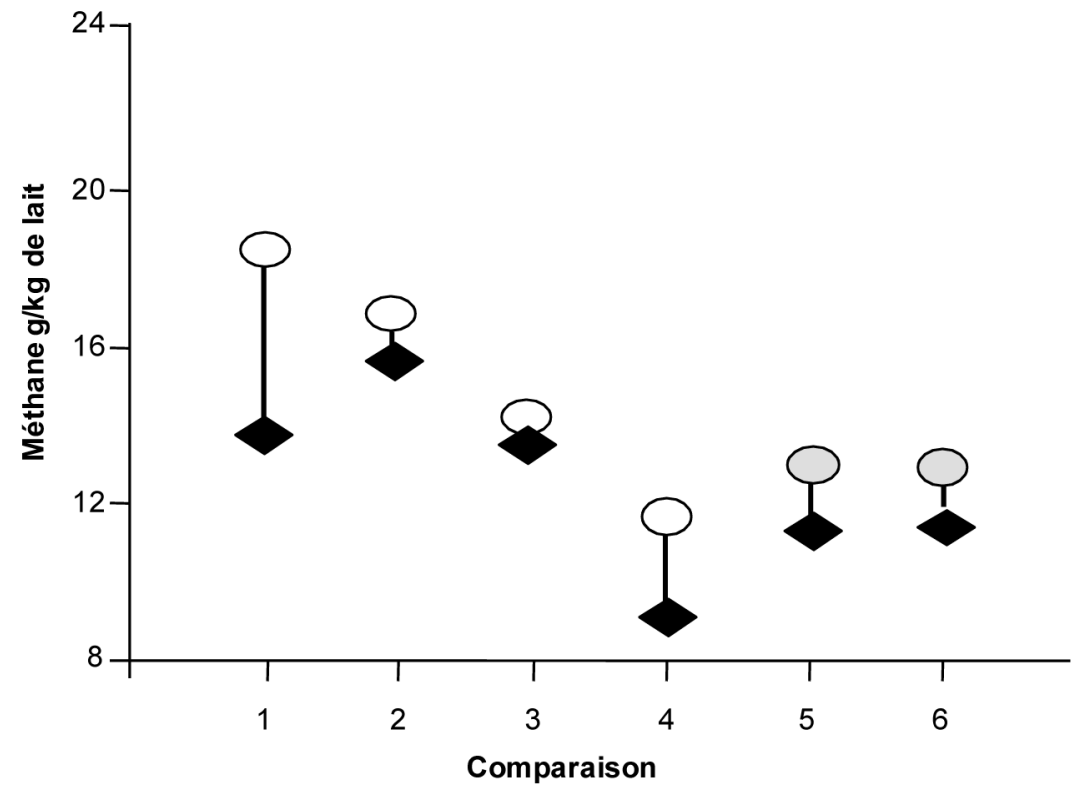

Ensilage de maïs

Foin

Ensilage d'herbe

matière organique, mais aussi à la teneur en NDF du fourrage, alors que ces deux facteurs varient en sens inverse (Archimède et al 2011). Ce résultat rend compte du fait que plus il y a de constituants digestibles, plus il y a de substrats pour la méthanogenèse, et que, à même digestibilité, plus il y a de NDF, plus la voie de l'acétate, et donc la méthanogenèse, sont favorisées. Il faut toutefois remarquer que la plage de variation des proportions d'AGV est plus faible entre fourrages qu'entre rations mixtes. Des comparaisons directes ont d'ailleurs donné des résultats contradictoires. Robertson et Waghorn (2002) ont observé que la production de méthane augmentait avec le stade de maturité du fourrage, alors que PinaresPatiño et al (2003) n'ont pas observé de variation de production de méthane en pourcentage de l'énergie brute ou de la matière organique ingérée. Par ailleurs, Kirkpatrick et Steen (1999) n'ont pas observé de différence de production de méthane en proportion de l'énergie brute ingérée entre un fourrage vert et un ensilage récoltés simultanément, ce qui est logique dans la mesure où le processus d'ensilage modifie peu la teneur en parois et la digestibilité.

La méta-analyse d'Archimède et al (2011) n'a pas mis en évidence de différence d'émission de méthane, in vivo, entre graminées et légumineuses tempérées. Toutefois, certains travaux suggèrent que les légumineuses sont à l'origine d'une émission de méthane entérique plus faible que les graminées. McCaughey et al (1999) ont observé une diminution de $10 \%$ de la production de méthane par kg de gain de poids sur des bovins à viande au pâturage, lorsque les graminées étaient remplacées par un mélange graminées-luzerne. Un tel effet n'a pas été relevé avec du trèfle blanc ou violet (Beever et al 1985, Van Dorland et al 2007, Hammond et al 2011); par ailleurs Carulla et al (2005) et Niderkorn et al (2011) n'ont observé aucun effet significatif de la luzerne ou du trèfle violet apporté en substitution à du ray-grass sur l'émission de méthane par $\mathrm{kg}$ de MS, toujours lors d'essais in vivo. L'effet de la luzerne parfois observé pourrait laisser penser que la réduction de méthane serait due à la richesse de la luzerne en malate, un acide organique contribuant à réduire la production de méthane (cf. § 4.3), ou à certains métabolites secondaires comme les saponines (cf. § 3.2). Toutefois, l'absence d'effet de la substitution de luzerne déshydratée à du tourteau de soja à raison de $30 \%$ de la ration (Doreau et al non publié) ne plaide pas pour un «effet luzerne» spécifique. Il faut toutefois noter que l'introduction de légumineuses dans la ration tend à réduire les émissions de protoxyde d'azote.
La méta-analyse d'Archimède et al (2011) a montré que les graminées ayant une photosynthèse en $\mathrm{C}^{2}$, caractéristique des climats chauds, induisent une production de méthane supérieure de 10 à $17 \%$ à celle des graminées ayant une photosynthèse en $\mathrm{C} 3$, caractéristique des climats tempérés, à même digestibilité, teneur en NDF et niveau d'ingestion. Les raisons en sont encore inexpliquées. Inversement, les légumineuses à photosynthèse en $\mathrm{C} 4$ induisent une production de méthane inférieure aux légumineuses à photosynthèse en $\mathrm{C} 3$, mais c'est très probablement dû à la proportion de tannins en moyenne supérieure dans les légumineuses tropicales. La présence de tannins contribue en effet à la réduction des émissions de méthane ; leur rôle sera détaillé dans le paragraphe 3.1 .

\section{3 / Apport de lipides}

L'enrichissement en lipides de la ration est une voie nutritionnelle prometteuse pour réduire les émissions de méthane. La raison la plus évidente est que les lipides, quels qu'ils soient, ne fournissent pas de substrat pour la production de méthane dans le rumen, contrairement à la fermentation des glucides auxquels ils se substituent. En outre, les actions sur les populations microbiennes décrites précédemment renforcent cet effet anti-méthanogène. La réduction de la production de méthane par apport de lipides a été quantifiée dans des équations proposées par GigerReverdin et al (2003) et Eugène et al (2008) pour les vaches laitières, et qui font respectivement état de diminutions moyennes de 2,2 et $1,7 \%$ de la production de méthane par $\mathrm{kg}$ de MS ingérée lorsque la teneur en lipides augmente d'un point, c'est à dire de $1 \%$ dans la $\mathrm{MS}$ de la ration. Dans l'étude d'Eugène et al (2008), cette diminution est non significative, mais elle l'est lorsque le méthane est exprimé par rapport à l'énergie brute ingérée. Dans une revue basée sur 17 études, Beauchemin et al (2008) rapportent une diminution de production de méthane par $\mathrm{kg}$ de MS ingérée de $5,6 \%$ par point de lipides supplémentaires dans la ration pour différentes espèces de ruminants. Dans une revue de synthèse, récapitulant 67 régimes enrichis en lipides tirés de 28 publications, Martin et al (2010) ont observé une diminution moyenne de $3,8 \%$ de méthane par kg de MS ingérée, par point de lipides supplémentaires. Une mise à jour de cette base de données a été réalisée; elle comprend maintenant 82 régimes enrichis en lipides tirés de

2 Les plantes fixent le $\mathrm{CO}_{2}$ lors de la photosynthèse par différentes voies métaboliques ; les deux principales sont dites en $\mathrm{C} 3$ et en $\mathrm{C} 4$, selon que la fixation de $\mathrm{CO}_{2}$ produit un composé à 3 ou 4 atomes de carbone. Les plantes fourragères de zone tempérée ont en général une photosynthèse en C3, le maïs et les plantes fourragères tropicales ont une photosynthèse en $\mathrm{C} 4$. 
Figure 3. Effet de la supplémentation lipidique sur la production de méthane, en fonction des AG majeurs de la source lipidique : revue de la bibliographie.

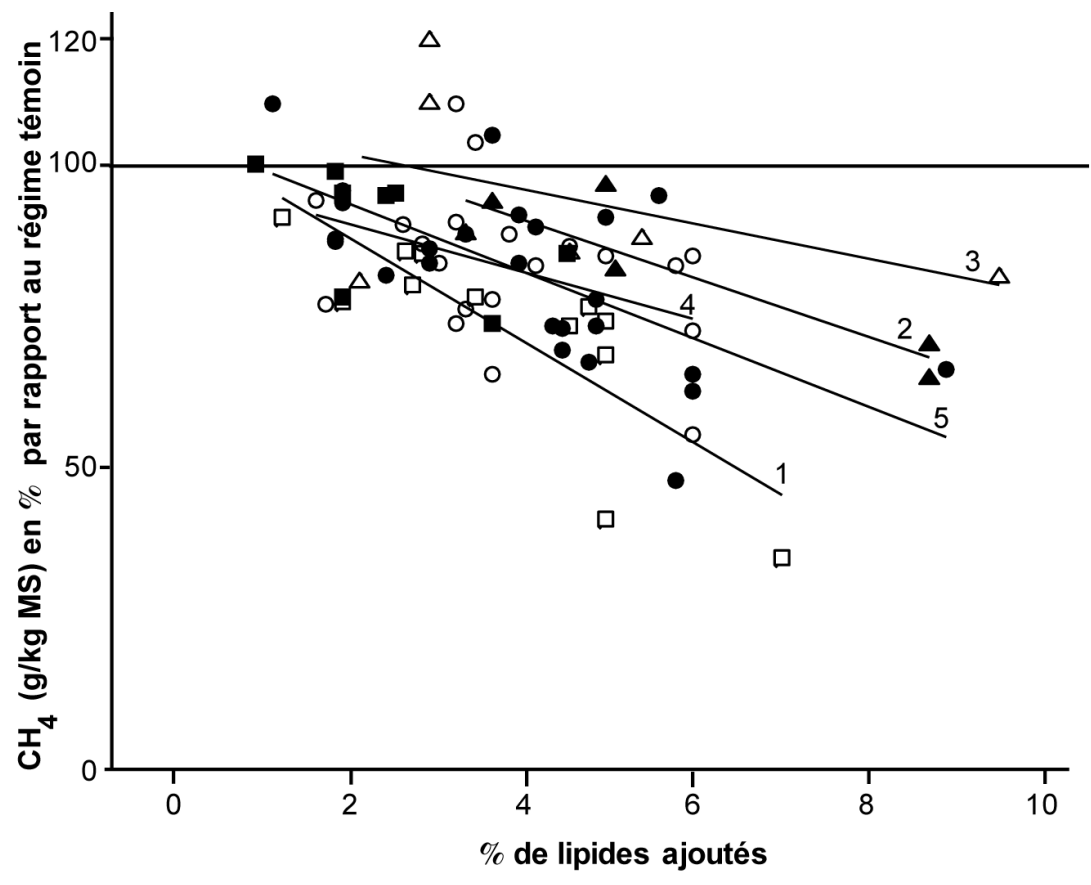

Nature de l'acide gras majoritaire : $\square$ 1-Chaîne moyenne; $\mathbf{\Delta} 2$ - Palmitique, Stéarique ; $\triangle$ 3-Oléique ; $\bigcirc$ 4- Linoléique; 5-Linolénique; Divers

33 publications. Cette synthèse (figure 3) montre en outre que la diminution moyenne de la production de méthane dépend de la nature des AG. Elle est la plus importante pour les $\mathrm{AG}$ à chaîne moyenne (apportés par le coprah, 8,5\% par point de lipides supplémentaires), puis pour l'acide linolénique (apporté par le lin, 5,6\% par point de lipides supplémentaires), puis pour l'acide linoléique (apporté par le tournesol et le soja, 4,0\% par point de lipides supplémentaires). Les AG mono-insaturés comme l'acide oléique (apporté par le colza), les AG saturés, palmitique et stéarique (savons de calcium d'huile de palme, graisses cristallisées) ont un effet plus limité. La figure 3 montre que dans un nombre limité d'essais l'apport de lipides entraîne un léger accroissement de la production de méthane par kg de MS, peut-être en raison d'interactions avec le reste de la ration. Très récemment, Grainger et Beauchemin (2011) ont étudié par méta-analyse 21 publications regroupant 92 traitements (60 enrichis en lipides et 30 témoins) et ont mis en évidence une diminution de $1,0 \mathrm{~g}$ de méthane par $\mathrm{kg}$ de $\mathrm{MS}$ ingérée (approximativement 4\%) par point de lipides supplémentaires. Mais ces auteurs n'ont détecté, avec une méthodologie différente de la nôtre, aucun effet significatif de la source de lipides, bien qu'ayant pris en compte l'effet essai, probablement en raison de la forte variabilité de réponse de la production de méthane à l'apport lipidique.

Cette forte variabilité se traduit par le fait que la comparaison directe entre sources de lipides donne des résultats contradictoires in vivo. Différents essais ont été récapitulés par Martin et al (2010). La comparaison des trois types d'AG les plus fréquemment testés (oléique, linoléique et linolénique) donne des résultats variables d'un essai à l'autre. Les connaissances actuelles sur l'effet des différentes sources de lipides sur les populations microbiennes du rumen (cf. Popova et al 2011) ne permettent pas d'expliquer ces divergences. L'effet des AG à 20 et 22 carbones sur la production de méthane est encore mal connu : il est très dépresseur in vitro (Fievez et al 2007) mais in vivo, l'huile de poisson, en association avec d'autres sources de lipides, n'a qu'un effet mineur (Woodward et al 2006). Des études complémentaires seraient nécessaires, peut-être avec des micro-algues dont l'utilisation est a priori plus acceptable que celle des huiles d'origine animale.

De nombreux essais ont été réalisés avec le lin. Parfois, il n'y a pas eu de réponse de la production de méthane (van Zijderveld et al 2011), parfois des réponses très fortes à dose élevée : Martin et al (2008) ont montré une diminution de la production de méthane de plus de 50\% avec un apport de 5,8\% d'huile de lin. La réponse à des doses croissantes de graine de lin extrudée est faible pour des apports modérés (diminution de production journalière de 6 à $15 \%$ pour $2 \%$ de lipides ajoutés), et plus prononcée pour des doses élevées (diminution de production journalière de 40 à $42 \%$ pour $6 \%$ de lipides ajoutés) (Martin et al 2009). L'emploi du lin pour réduire la production de méthane s'accompagne d'un effet plutôt bénéfique sur la qualité nutritionnelle du lait et de la viande, en raison d'une légère augmentation des $A G$ en oméga 3 (acide linolénique). Toutefois, un apport excessif de lin peut se traduire par une limitation du niveau d'ingestion des animaux, un accroissement dans les produits de certains AG monoinsaturés trans (Doreau et al 2011a), et par une tendance à la péroxydation des lipides. Dans l'état actuel des connaissances, un apport de lipides du lin de l'ordre de 2 à $3 \%$ peut se révéler efficace pour réduire la méthanogenèse (Martin et al 2011). Quand aux AG à chaîne moyenne qui ont des effets marqués sur la méthanogenèse, leur emploi devrait être freiné par leur effet plutôt négatif sur la «valeur santé» des produits animaux, et peut-être par une réduction de digestibilité des rations dans certains cas.

Parmi les sources de lipides, on peut citer les drêches de maïs de distillerie, sous-produit de la fabrication d'éthanol. Ce produit ne contient qu'environ $10 \%$ d'AG, mais comme il peut être incorporé en quantités élevées dans la ration, il constitue un moyen d'enrichir la ration en lipides ; McGinn et al (2009) ont obtenu sur des bovins à viande une baisse de la production de méthane de $16 \%$ en remplaçant l'orge (35\% de la ration) par des drêches de maïs sèches avec solubles ${ }^{3}$. D'autres sous-produits d'agrocarburants, comme les tourteaux gras de colza à $10 \%$ de lipides, ont le même effet (Moate et al 2011).

L'effet de l'apport lipidique sur la méthanogenèse peut dépendre de la composition de la ration, mais les données sont fragmentaires et contradictoires. La baisse de méthane avec des apports croissants de graine de lin est plus prononcée en pourcentage avec une ration à base de foin qu'avec une ration à base d'ensilage de maïs (Martin et al 2009), et inversement plus prononcée avec de

\footnotetext{
${ }^{3}$ Sous-produit de la fabrication d'éthanol à partir de céréales, en anglais DDGS (Dry Distillers Grains with Solubles).
} 
l'ensilage d'orge qu'avec du foin (Chung et al 2011a); avec des AG à chaîne moyenne elle est plus prononcée avec une ration riche en concentré qu'avec une ration riche en fourrage (Lovett et al 2003, Machmüller et al 2003).

Peu d'essais à long terme ont été réalisés. Grainger et Beauchemin (2011) ont récapitulé 7 essais à moyen et long terme avec différentes sources de lipides, dont seulement 3 ont duré plus de 3 mois, et tirent la conclusion que l'effet des lipides se maintient dans le temps. Holter et al (1992) et Grainger et al (2010) avec des graines de coton distribuées à des vaches respectivement pendant 16 et 12 semaines ont même trouvé que l'effet des lipides s'accentuait dans le temps. Enfin, Martin et al (2011) ont établi qu'après une année de complémentation en graine de lin de la ration de vaches laitières, la production de méthane était inférieure à celle de la ration non supplémentée. Au contraire, Eugène et al (2011) ont observé avec des taurillons engraissés durant 6 mois que la réduction de la production de méthane par $\mathrm{kg}$ de gain de poids d'une ration concentrée riche en amidon et lipides en comparaison avec une ration concentrée riche en fibres, était moins prononcée au cours du temps, mais l'effet peut être attribué aussi bien aux lipides qu'à l'amidon. Toutefois ces différents essais considérés ensemble, laissent penser que l'effet des lipides polyinsaturés sur la production de méthane pourrait être permanent.

\section{3 / Plantes spécifiques et huiles essentielles}

Un nombre considérable de mesures de la production de méthane a été réalisé in vitro avec des plantes très diverses, choisies parfois pour leur richesse connue en métabolites secondaires, parfois au hasard. L'essai le plus emblématique est celui de Bodas et al (2008) qui ont testé 450 plantes ; Garcia-Gonzalez et al (2008) dans la même équipe ont réalisé un essai similaire sur 158 plantes, certaines étant communes avec l'essai précédent. $\mathrm{Au}$ total, moins de 10 plantes ou extraits ont fortement réduit la production de méthane sans effet négatif sur les fermentations, en particulier deux variétés de rhubarbe. L'utilisation potentielle de telles plantes, si elles se montraient efficaces, est bien sûr tributaire de leur aptitude à la culture ainsi que de leur absence de toxicité. Les plantes tropicales, riches en métabolites secondaires, sont également largement étudiées (Soliva et al 2008, Jayanegara et al 2011a), mais seules certaines d'entre elles représen- tent une biomasse non négligeable, dans certains pays. Une récente revue a décrit les effets des principales plantes étudiées (Patra et Saxena 2010). Les métabolites secondaires les plus prometteurs sont les tannins et les saponines, mais bien d'autres constituants sont susceptibles de réduire la production de méthane, et sont souvent présents dans des huiles essentielles.

\section{1 / Plantes riches en tannins}

Les tannins sont connus depuis plusieurs années pour leur action inhibitrice sur la production de méthane entérique. Ceci vient d'être confirmé par une récente méta-analyse qui récapitule 15 essais in vitro et 15 essais in vivo (Jayanegara et al 2011a). Cet effet pourrait être lié en particulier à la forte réduction des Archaea méthanogènes (Popova et al 2011). Les tannins peuvent être consommés dans certains fourrages distribués aux animaux à plus ou moins grande échelle, ou sous forme d'extraits ajoutés à la ration. Sous le nom générique de tannins, on range deux familles très différentes de composés : les tannins condensés, dont l'effet négatif sur la production de méthane a été très bien étudié, et les tannins hydrolysables, dont ce même effet est moins connu, mais a été mis en évidence récemment par Jayanegara et al (2011b). L'introduction dans la ration de tannins condensés, mais aussi de tannins hydrolysables, entraîne d'autres effets qui peuvent être favorables comme la réduction de la dégradation des protéines dans le rumen ou la possible réduction de l'hydrogénation des AG, ou défavorables, comme la diminution fréquente de la digestibilité de la matière organique (Waghorn 2008). Il est donc important de s'assurer que la réduction de la production de méthane ne s'accompagne pas d'un effet dépressif sur la digestibilité ; des essais avec des taux d'incorporation croissants d'une même source de tannins permettraient certainement de répondre à cette interrogation. De nombreuses légumineuses tropicales sont riches en tannins, en particulier des légumineuses arbustives comme Leucaena leucocephala, qui constituent une ressource alimentaire non négligeable dans certains pays. Bien que la nature et la structure biochimique des tannins varie d'une plante à l'autre, Jayanegara et al (2011b) ont montré une relation négative entre l'émission de méthane in vitro et les teneurs en tannins condensés, mais aussi en tannins hydrolysables; toutefois les plantes ayant la meilleure valeur nutritive entraînent également une production de méthane élevée, peut-être parce qu'elles sont plus riches en glucides fermentescibles. Bien que les plantes à tannins soient plus répandues dans les zones tropicales, elles sont utilisées dans les zones tempérées (lotier, sainfoin) de même que des extraits de tannins ajoutés à la ration.

Carulla et al (2005) ont montré que l'extrait de tannins d'écorce d'acacia noir (A. Mearnsii) réduisait la production de méthane par des moutons à un taux d'incorporation de $2,5 \%$ de la MS, avec toutefois une légère diminution de digestibilité compensée par une légère augmentation d'ingestion. Cet effet a été confirmé par Grainger et al (2009) avec des vaches laitières au pâturage à un taux d'incorporation de $2 \%$ de la MS, puis par Staerfl et al (2012) dans un essai à long terme avec des taurillons, à un taux d'incorporation de $3 \%$ de la MS ; la digestibilité des parois était également réduite, mais sans conséquence sur le gain de poids des animaux. En revanche, Beauchemin et al (2007) n'ont pas trouvé d'effet de l'extrait de tannin de quebracho distribué à raison de $2 \%$ de la MS, de même que Sliwinski et al (2002) avec 1 à $2 \%$ de tannins de châtaignier dans la MS. Il est difficile de savoir si ces différences doivent être imputées à la nature des tannins ou aux conditions expérimentales.

Parmi les légumineuses à tannins cultivées en régions tempérées, le lotier corniculé (Lotus corniculatus) a été largement étudié, principalement en Nouvelle-Zélande, et son effet sur la diminution de méthane semble clair. Par exemple, les émissions de méthane par $\mathrm{kg}$ de MS ou $\mathrm{kg}$ de lait par des vaches laitières sont plus faibles avec le lotier corniculé qu'avec le ray-grass, et cet effet est dû aux tannins, car il est supprimé lorsque les tannins sont inactivés par le polyéthylène glycol, molécule se fixant sur les tannins (Woodward et al 2004). Un effet similaire a été observé avec des vaches laitières sur une légumineuse à tannins du Sud de l'Europe, le sainfoin d'Italie (Hedysarum coronarium) (Woodward et al 2002); en revanche, on manque de références in vivo sur le sainfoin (Onobrychis sp.).

\section{2 / Plantes riches en saponines}

Les saponines sont des glycosides présents dans de nombreuses plantes. Ils diminuent la dégradation des protéines dans le rumen et favorisent la synthèse de protéines microbiennes, ce qui limite la quantité d'hydrogène disponible pour la méthanogenèse. Les saponines réduisent la population de protozoaires (Newbold et Rode 2006) ; l'effet sur les différentes populations microbiennes du rumen dépend du type de ration et a été détaillé par Popova et al (2011). In vitro, l'effet négatif des saponines sur la méthanogenèse a été montré avec différentes plantes, mais il n'est pas sys- 
tématique. Toutefois, dans certains cas, avec des doses élevées de saponines, des diminutions de $25 \%$ ont été mises en évidence. C'est le cas d'un essai de Holtshausen et al (2009) avec un niveau d'incorporation de Yucca schidigeria et de Quillaja saponaria dans le fermenteur correspondant à $4,5 \mathrm{~g} / \mathrm{kg}$ de $\mathrm{MS}$; toutefois à ces niveaux d'incorporation la digestibilité des parois in vitro était fortement réduite.

Les essais in vivo sont en nombre plus limité. Les sources de saponines les plus étudiées sont celles qui ont donné des résultats concluants dans des tests in vitro et qui sont les deux principales sources commercialisées, Yucca schidigeria et Quillaja saponaria. Les diminutions de méthane ont été soit nulles, parfois en raison de doses très faibles, soit de l'ordre de 5 à $10 \%$ et non significatives, avec des doses voisines de $1 \mathrm{~g} / \mathrm{kg}$ de MS ingérée avec des moutons pendant de courtes périodes (Pen et al 2007), avec des vaches laitières après des périodes d'adaptation de 4 semaines (Holtshausen et al 2009), et curieusement de l'ordre de $15 \%$ et significative dans un essai de courte durée de Wang et al (2009) avec une dose de $0,1 \mathrm{~g} / \mathrm{kg}$ de MS. Par ailleurs, deux essais sur moutons avec des périodes expérimentales de 3 semaines ont mis en évidence des diminutions de la production de méthane significatives d'environ $10 \%$, l'un avec des fruits de Sapindus saponaria (Hess et al 2004) et l'autre avec des saponines de thé (Zhou et al 2011). Un effet dépressif de $27 \%$ des saponines de thé a même été observé par Mao et al (2010) sur moutons après 2 mois d'adaptation. Le nombre de données disponibles et concordantes est toutefois trop faible pour qu'une conclusion puisse être tirée sur les possibilités d'emploi de certaines plantes riches en saponines comme agents permettant de réduire la production de méthane.

\section{3 / Huiles essentielles et autres extraits de plantes}

L'intérêt des huiles essentielles pour réduire la méthanogenèse a fait l'objet de la revue récente de Benchaar et Greathead (2011). Ces auteurs mentionnent tout d'abord la très grande variabilité de composition en huiles essentielles d'une même plante, en fonction entre autres de leur région d'origine. Ceci rend l'interprétation des résultats expérimentaux difficile lorsque des extraits de plantes sont analysés pour leurs effets sur les fermentations ruminales. En outre, la multiplicité des molécules actives au niveau de la population microbienne du rumen se traduit par des effets inhibiteurs sur les Archaea méthanogènes et sur certaines espèces bactériennes, ce qui peut réduire l'intensité des fermentations. Parmi les composés dont l'action négative sur la production de méthane a été montrée in vitro, la réponse à des doses croissantes a été étudiée pour le carvacrol, le thymol, l'eugénol et le cinnamaldéhyde, ainsi que pour les huiles plus complexes de thym, de cannelle et d'origan par Macheboeuf et al (2008) et par Benchaar et Chiquette (non publié, cités par Benchaar et Greathead 2011). Les huiles d'origan et de thym contiennent du carvacrol et du thymol, et en moindre quantité des terpènes qui sont leurs précurseurs, le $\gamma$-terpinène et le p-cymène. Macheboeuf et al (2008) ont montré que ces huiles ont des effets plus marqués que les molécules qui les composent, suggérant des effets additifs ou même synergiques. Il en serait de même pour l'huile de cannelle et ses composants, le cinnamaldéhyde qui est le composé prédominant et l'eugénol. A notre connaissance, il n'existe pas d'essai in vivo testant l'efficacité de ces molécules pour réduire les émissions de méthane, sans perturber l'ingestion ou la digestion. Par ailleurs, Mohammed et al (2004) ont montré que l'huile de raifort protégée pour éviter des chutes d'ingestion a réduit la production de méthane de près de $20 \%$ chez des bouvillons ; cet essai de courte durée n'a toutefois pas été confirmé.

L'association de deux ou plusieurs huiles essentielles pourrait accroître les chances de succès d'une stratégie de réduction de la production de méthane. Par ailleurs, Beauchemin et McGinn (2006) ont montré l'inefficacité d'un mélange commercial d'huiles essentielles et d'épices.

L'extrait d'ail a été souvent montré comme très efficace pour réduire la production de méthane in vitro, en raison de la présence de composés organosulfurés. In vivo, les résultats sont décevants. Klevenhusen et al (2011) et van Zijderveld et al (2011) n'ont pas observé de diminution de la production de méthane avec l'extrait d'ail ou son composé qui semble le plus efficace, le bisulfure de diallyl, qui inhiberait spécifiquement les Archaea méthanogènes; toutefois le bisulfure de diallyl aurait un effet positif sur la digestibilité. L'absence d'effet d'une supplémentation avec des bulbes d'ail sur la production de méthane a aussi été observée par Patra et al (2011) avec des moutons, et par Staerfl et al (2012) avec des taurillons. Si l'effet de l'extrait d'ail s'avérait significatif dans le futur, son usage pourrait être limité à la production de viande, en raison de l'apparition d'odeurs dans le lait.
Parmi les autres extraits de plantes dont l'effet négatif sur la production de méthane a été montré in vivo, on peut également citer la noix de cajou, dont l'effet avait été préalablement montré in vitro. Selon Shinkai et al (2010) un supplément de $23 \mathrm{~g} / \mathrm{j}$ de noix de cajou à des vaches laitières a réduit de $20 \%$ la production de méthane; mais très peu de détails sont disponibles sur cet essai. Un essai récent (Tekippe et al 2011) a montré un effet très net de la feuille d'origan $(500 \mathrm{~g} / \mathrm{j})$ sur la production de méthane de vaches laitières $(-40 \%)$ dans un essai de courte durée (périodes de 3 semaines); toutefois les auteurs suggèrent que la méthodologie employée a pu surestimer la réduction de méthane. Par ailleurs, Wang et al (2009) ont observé une diminution de la production de méthane de $12 \%$ après addition de 250 $\mathrm{mg} / \mathrm{j}$ d'un extrait d'origan, commercialisé sous le nom de ropadiar ${ }^{\circledR}$, dans la ration de moutons, dans un essai de courte durée (périodes de 2 semaines). Il convient d'être prudent, car l'effet in vitro de l'origan sur la méthanogenèse n’est pas systématique.

En conclusion, il est difficile d'extrapoler des résultats prometteurs in vitro aux conditions in vivo, et beaucoup de travail reste à faire pour démontrer l'efficacité de certaines huiles essentielles ; jusqu'à présent aucun essai à long terme n’a été réalisé.

\section{4 / Additifs et biotechnolo- gies}

Dans cette partie ne sont pas traitées les techniques visant à réduire spécifiquement les méthanogènes (vaccination ou anticorps, composés chimiques toxiques pour les méthanogènes comme le chloroforme, le bromoéthylsulfonate et le bromochlorométhane) dont le rôle et l'efficacité sont détaillés dans l'article de Popova et al (2011).

\section{1 / Addition de probiotiques}

Les levures vivantes, exclusivement représentées par différentes souches de Saccharomyces cerevisiae, sont utilisées depuis plusieurs années comme additifs dans la ration des ruminants essentiellement pour stimuler la dégradation des fibres, pour limiter la dégradation des protéines ou pour prévenir l'acidose (Chaucheyras-Durand et al 2008). L'addition des souches commercialisées n'a pas montré jusqu'à présent d'effet sur la production de méthane (Doreau et Jouany 1998, entre autres), mais il n'y a pas de souches commercialisées qui soient spécifiquement développées pour réduire la méthanogenèse. Certaines souches ont montré in vitro 
une réduction de la production de méthane (Newbold et Rode 2006), et une première publication fait état d'une tendance non significative à une réduction de la production de méthane in vivo chez la vache avec une souche sélectionnée (Chung et al 2011b).

L'incorporation de bactéries probiotiques comme additif se développe. Pour les ruminants adultes, ces bactéries sont destinées à prévenir l'acidose, ou à limiter le portage des pathogènes potentiels tels que certaines souches d'Escherischia coli et Salmonella spp. Les bactéries les plus communément utilisées sont des utilisatrices de lactate qui accroissent la production de propionate, et des bactéries lactiques. Un travail récent dans notre laboratoire, utilisant une association de bactéries probiotiques, a permis, pour la première fois à notre connaissance, de diminuer significativement la production de méthane chez la vache, sans modification de la production laitière, dans un essai de courte durée (données non publiées). $\mathrm{Ce}$ résultat prometteur reste à confirmer.

L'utilisation de l'hydrogène par la voie de l'acétogenèse existe chez l'Homme et différentes espèces animales. Dans le rumen, les bactéries acétogènes ne sont pas compétitives avec les Archaea méthanogènes pour l'utilisation de l'hydrogène. Récemment, il a été montré in vitro que de telles bactéries de l'intestin du kangourou étaient compétitives vis-à-vis des Archaea méthanogènes du rumen (Klieve et Joblin 2007). Cet essai a eu une forte publicité médiatique, mais la culture de telles bactéries et leur implantation dans le rumen restent à faire. Il existe donc une voie d'exploration, mais il est probable que des probiotiques contenant des bactéries acétogènes puissent être efficaces lorsqu'une stratégie de suppression des méthanogènes est appliquée conjointement (Joblin 1999).

L'effet de microorganismes exogènes sans activité probiotique a également été testé. Récemment une culture fongique de Monascus sp. s'est révélée efficace in vivo et a conduit à une réduction de la production de méthane de $30 \%$ chez le mouton après 2 semaines d'apport. Ce résultat, dû à différents métabolites de ce champignon, s'explique par une orientation des fermentations vers le propionate et une baisse de la population de méthanogènes (Morgavi et al 2010b). L'applicabilité de cette technique, et la confirmation de l'effet à long terme restent à montrer.

\section{2 / Antibiotiques ionophores}

Les antibiotiques ionophores, principalement le «monensin» et le «lasalocide», sont une catégorie d'antibiotiques dotés de propriétés spécifiques (stimulation du transport actif des cations et réduction de la production d'ATP) qui se traduisent par une toxicité vis-à-vis des bactéries à gram positif (Jouany 1994). Ils sont interdits dans l'Union Européenne depuis 2006, mais sont toutefois toujours cités comme un moyen possible de réduire les émissions de méthane (Moran et al 2011). Leur emploi chez le ruminant entraîne à court terme une diminution de la production de méthane de 0 à $30 \%$. D'après la revue de Beauchemin et al (2008), l'effet dépendrait du niveau d'incorporation; la production de méthane diminuerait en moyenne de 3 à $8 \%$ par $\mathrm{kg}$ de MS ingérée à des doses élevées (20 à 35 $\mathrm{mg}$ par kg de MS ingérée). Cet effet est relié à l'orientation des fermentations ruminales vers l'acide propionique et à une stimulation des bactéries à gram négatif ; en outre la population de protozoaires est réduite, mais celle des méthanogènes n'est pas affectée. A long terme, l'effet a été confirmé dans des essais nord-américains par Odongo et al (2007) avec des vaches laitières (-7\% après 6 mois d'application) mais pas par Guan et al (2006) avec des bouvillons : la baisse de production de méthane observée durant le premier mois ne s'est pas maintenue ultérieurement, en lien avec une chute transitoire de la population de protozoaires, qui se sont adaptés aux ionophores. Il est à noter qu'un autre antibiotique promoteur de croissance, la flavomycine, a montré une certaine efficacité in vivo pour réduire la production de méthane dans un essai de courte durée (Wang et al 2009), mais son emploi est interdit de la même manière que celui des ionophores.

\section{3 / Acides organiques}

Les acides organiques sont des constituants mineurs de certaines plantes; le cas de la luzerne a été évoqué précédemment. Dans le rumen, ils sont convertis par des bactéries en succinate avec consommation d'hydrogène venant du dihydrogène ou du formate; le succinate est à son tour converti en propionate. Trois acides organiques de synthèse ont été étudiés in vitro pour leur effet négatif sur la production de méthane, le malate, le fumarate et l'acrylate, et les deux premiers l'ont été in vivo. In vitro, la réduction de la méthanogenèse n'est pas systématique ; lorsque l'effet est significatif, celui du fumarate est plus important que celui du malate, et celui des formes acides plus important que celui des sels (Newbold et al 2005). Par ailleurs, Jouany et al (2008) ont observé en fermenteur de type «batch» ${ }^{4}$ que l'association entre acide malique et huile de tournesol réduisait la production de méthane, alors que chacun de ces composés pris seul n'avait pas d'effet. Curieusement, l'effet des acides organiques sur la méthanogenèse est souvent plus important in vivo ou en fermenteur continu que dans des systèmes in vitro simples de type «batch». Ceci peut être dû à une compétitivité plus grande des bactéries utilisant les acides organiques par rapport aux méthanogènes dans des systèmes complexes. Toutefois, l'effet le plus spectaculaire a été obtenu avec des agneaux en croissance par Wallace et al (2006) avec l'incorporation dans la ration de $10 \%$ de fumarate protégé de la dégradation dans le rumen par encapsulation, la production de méthane étant réduite jusqu'à $75 \%$ après 2 mois sur ce régime. Cet effet n'a pas été reproduit ultérieurement dans un essai sur vaches laitières (McCourt et al 2008).

Lorsqu'on calcule par stoechiométrie l'amplitude théorique de diminution de la production de méthane liée à l'effet «puits d'hydrogène» des acides organiques, on se rend compte que l'effet strict de l'utilisation d'hydrogène lors du métabolisme des acides organiques ne suffit pas à expliquer les réponses parfois observées: une distribution de $2 \mathrm{~kg}$ de fumarate par jour à une vache laitière ne diminuerait la production de méthane de seulement $10 \%$ (Newbold et Rode 2006). Par ailleurs, Ungerfeld et Forster (2011) ont montré à partir de données in vitro que seulement la moitié du malate était convertie en propionate. D'autres mécanismes conduisant à la réduction de la production de méthane sont donc à rechercher. Même si les acides organiques se montraient efficaces sur le long terme pour réduire la méthanogenèse, il y a plusieurs freins à leur utilisation pratique : la possible restriction de l'usage de molécules de synthèse en alimentation animale, le fait que des quantités importantes soient nécessaires, ce qui peut entraîner un problème pour l'image des produits et, en corollaire, leur coût élevé.

Le glycérol, composé disponible en grande quantité lors de la production d'agrocarburants, n'est pas un acide organique, mais est converti en propionate, et son inclusion dans la ration en

\footnotetext{
${ }^{4}$ Les fermenteurs de type «batch» sont des systèmes simples qui permettent l'incubation de substrats avec un contenu de rumen en milieu clos pendant une période limitée (souvent $24 \mathrm{~h}$ ). A l'opposé, les fermenteurs continus sont alimentés en substrats et les produits de fermentation sont évacués, pendant plusieurs jours.
} 
substitution à d'autres sources énergétiques pourrait diminuer la production de méthane. Deux récents essais in vitro n'ont pas montré d'effet significatif de cette molécule : ainsi Avila et al (2011) n'ont toutefois pas observé de différence de production de méthane lorsque du glycérol était substitué à de l'orge.

\section{4 / Sulfates et nitrates}

Les voies métaboliques alternatives de la méthanogenèse telles que la réduction des nitrates et des sulfates ont été décrites par Morgavi et al (2010a). Brièvement, certaines bactéries sont capables d'oxyder l'hydrogène en utilisant des sulfates, des nitrates ou d'autres composés azotés; la proportion, normalement faible, de ces bactéries dans la microflore ruminale, augmente en présence de leur substrat de prédilection. L'utilisation de sulfates présente le risque de formation d'hydrogène sulfuré toxique, mais un essai in vitro récent a montré que les microorganismes sulfato-réducteurs pouvaient diminuer la production de méthane sans perturber la digestion et sans accroître la production d'hydrogène sulfuré (Paul et al 2011). La conversion de nitrates en ammoniac est compétitive vis-à-vis de la méthanogenèse. Pour cette raison, l'incorporation de nitrates dans la ration est envisagée. Ce procédé doit bien sûr être très maîtrisé, afin d'éviter la production de nitrites toxiques. Après de premiers essais concluants in vitro, un essai in vivo à court terme sur moutons a montré une réduction de production de méthane par addition de nitrates, de sulfates et de respectivement (Van Zijderveld et al ces deux composés de 32, 16 et $47 \%$

2010a) ; un second essai sur vaches laitières a montré l'efficacité à long terme de l'addition de nitrates sur la réduction de production de méthane (Van Zijderveld et al 2010b). Un brevet relatif à l'utilisation de sulfates et de nitrates dans la ration du ruminant a été déposé. Parallèlement, l'utilisation d'un autre composé azoté, le nitroéthane, a réduit à court terme la production de méthane in vivo (Brown et al 2011). L'utilisation de ce type de molécules se confrontera à l'acceptabilité de telles pratiques par le consommateur; bien qu'elles ne soient pas encore commercialisées en France, leur mise en marché est toutefois possible si elles se révèlent efficaces.

\section{5 / Effet du type d'animal}

\section{1 / Niveau de production}

L'augmentation du niveau de production des animaux entraîne une diminution de l'émission de méthane par $\mathrm{kg}$ de lait ou de viande. Cette diminution avec le niveau de production, très forte pour des productions laitières inférieures à $3000 \mathrm{~kg}$ de lait (Gerber et al 2011), donc essentiellement pour les bovins des pays en développement, est plus modérée au-delà (figure 4). Ce phénomène a plusieurs explications. Tout d'abord, la production de méthane par $\mathrm{kg}$ de MS ingérée diminue lorsque le niveau d'alimentation augmente, car le temps de séjour des aliments dans le rumen diminue (Pinares-Patiño et al 2007). Ensuite, comme cela a été précisé plus haut, aux niveaux d'ingestion élevés la méthanogenèse est réduite

Figure 4. Effet du niveau de production des vaches laitières sur les émissions de méthane par kg de lait.

Les cercles et le trait plein sont adaptés de Vermorel (1995) ; le trait pointillé constitue l'extrapolation de la courbe.

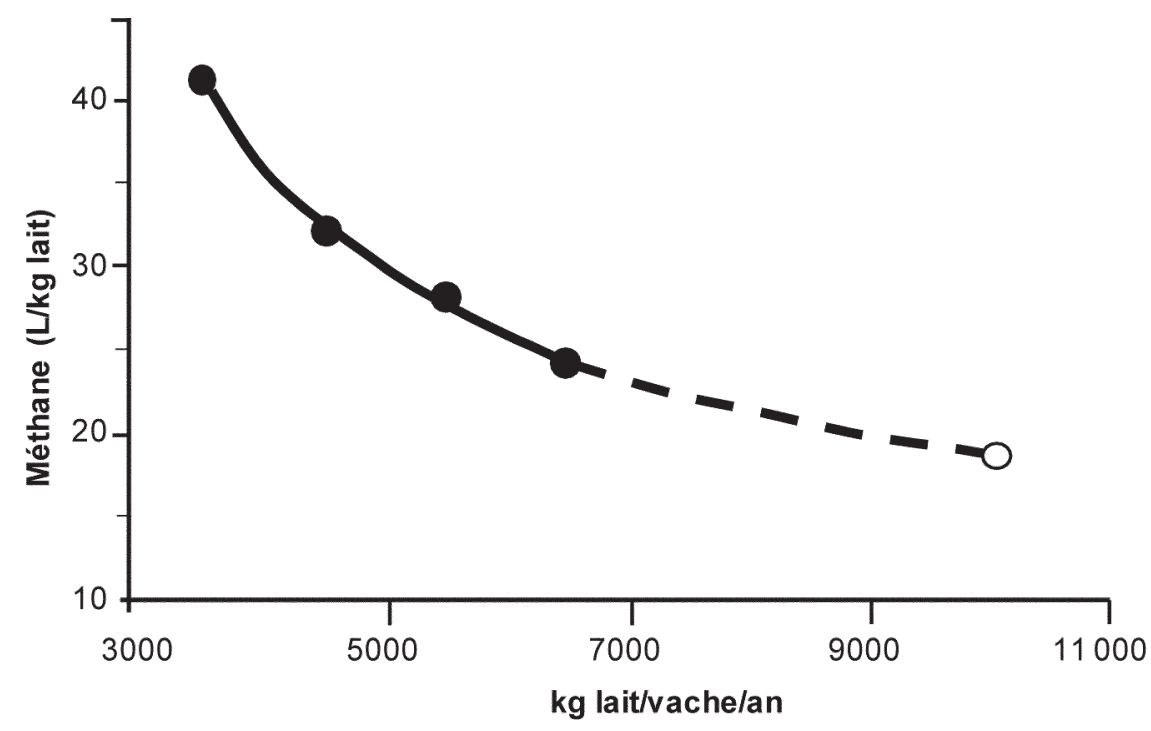

avec l'augmentation du pourcentage de concentré, qui est pratiquée lorsque le niveau de production s'élève. Enfin, lorsqu'on exprime l'émission de méthane par kg de lait ou de gain de poids, la part des besoins d'entretien dans les besoins totaux est plus faible quand le niveau de production s'élève, et par conséquent sur l'ensemble d'une lactation la production de méthane rapportée à l'unité de produit diminue. Il est à noter qu'une compensation existe au niveau de la carrière de l'animal, car les animaux forts producteurs ont une carrière plus courte et des performances de reproduction moins bonnes qui peuvent augmenter l'intervalle entre vêlages, ceci contribue, sur la vie de l'animal, à accroître la proportion des besoins liés aux périodes de croissance et de tarissement (Garnsworthy 2004).

L'accroissement du niveau de production a souvent été mentionné comme le levier d'action majeur pour réduire les émissions de méthane. Ceci doit être tempéré par le fait que l'augmentation de productivité animale s'accompagne souvent d'un accroissement de l'intensification et des intrants (fertilisants, concentrés achetés) et donc des émissions de protoxyde d'azote et de gaz carbonique par kg de produit. Ceci est illustré dans le texte de Dollé et al (2011) pour les conditions françaises. Une compensation partielle est également observée lorsqu'on compare des pays à productivité animale différente (Gerber et al 2011). Par ailleurs, les émissions de GES peuvent être rapportées à un territoire, et donc à une unité de surface, et dans ce cas les différences liées à la productivité sont plus faibles, même lorsqu'on prend en compte les surfaces des cultures utilisées pour l'alimentation animale.

\section{2 / Effets génétiques}

Depuis quelques années, les différences entre animaux dans la capacité de produire du méthane sont l'objet de nombreuses recherches. Les travaux n'ont pas montré d'effet du génotype, lorsque les animaux de différentes races étaient conduits de manière identique (Münger et Kreuzer 2008). Ceci est assez logique dans la mesure où la production de méthane dans le rumen est liée en priorité à l'écosystème microbien, et de manière secondaire à l'animal hôte ; celui-ci peut intervenir par le biais du temps de séjour des aliments dans le rumen, mais il y a peu d'effet de la race sur ce paramètre. Il existe en revanche des différences individuelles dans l'écosystème microbien pour une même ration, conduisant à des différences d'efficacité digestive (Zhou et al 2010), et donc de production de méthane. Une première méthode d'approche 
de l'existence d'effets génétiques est l'étude de la répétabilité des mesures de production de méthane. Différents auteurs, comme Pinares-Patiño et al (2007) et Vlaming et al (2008) qui ont mesuré le méthane produit par respectivement 9 et 4 vaches taries recevant des régimes contrastés, ont observé une répétabilité modérée, estimée par le rapport entre la variabilité mesurée entre animaux et la variabilité totale; le classement des animaux par ordre de production de méthane différait selon les régimes. Ces résultats peu encourageants peuvent s'expliquer par l'incertitude de la mesure du méthane par la technique du $\mathrm{SF}_{6}$, signalée par certains auteurs, ou par le fait que le changement de régime induisait des modifications différentes dans l'écosystème microbien d'un animal à l'autre. Un essai récent (Pinares-Patiño et al 2011) mené sur 105 moutons inclus dans un programme génétique a montré sur une première ration une répétabilité et une héritabilité non négligeables; dans un second temps les 10 animaux les plus forts et les 10 animaux les plus faibles producteurs de méthane ont été conservés, et les écarts ont été maintenus sur deux autres rations, et sont plus importants lorsque le pourcentage de concentré augmente. Il est probable que le choix d'animaux forts et faibles producteurs de méthane dans une population importante est la cause de ces résultats plus probants. La part du microbiote et de la physiologie digestive de l'animal hôte (transit, sécrétions salivaires, $\mathrm{pH} . .$. ) sur les productions de méthane de lignées divergentes reste à étudier.

Une seconde méthode d'approche sur animaux en production consiste à mesurer l'efficacité productive globale des animaux grâce au paramètre d'ingestion résiduelle «residual feed intake». L'ingestion résiduelle est la différence entre l'ingestion réelle et l'ingestion prévue par un modèle ou par régression. Les animaux ayant l'ingestion résiduelle la plus faible mangent moins pour un gain de poids équivalent; ils ont donc une efficacité productive globale, digestive et métabolique, supérieure, et ce critère devient l'objet de nombreuses études sur bovins en croissance. Nkrumah et al (2006) avec des bovins ayant des ingestions résiduelles différentes, et Hegarty et al (2007) avec des lignées obtenues par sélection divergente sur l'ingestion résiduelle, ont montré que pour un même gain de poids la production de méthane par jour (et donc par $\mathrm{kg}$ de gain de poids) était plus faible de $25 \%$ pour les bovins à faible ingestion résiduelle. Toutefois dans le premier essai les différences d'ingestion entre les deux lots étaient modérées, et la production de méthane par $\mathrm{kg}$ de MS ingérée était réduite de $8 \%$ pour les bovins à faible ingestion résiduelle, alors que dans le second, les différences d'ingestion étaient très fortes en raison de la sélection divergente, et la production de méthane par kg de MS ingérée était plus forte de $25 \%$ pour les bovins à faible ingestion résiduelle. Ces résultats montrent qu'il est intéressant d'utiliser des animaux à forte efficacité productive lorsqu'on raisonne en termes d'émission de méthane par $\mathrm{kg}$ de produit, mais que cela n'est pas dû à une diminution des productions quotidiennes de méthane. Par ailleurs, Jones et al (2011) ont montré que des vaches allaitantes différant par leur ingestion résiduelle émettaient la même quantité de méthane par jour sur un pâturage médiocre en gestation ou durant le tarissement, mais que le groupe ayant la plus faible ingestion résiduelle avait la plus faible émission de méthane sur de bons pâturages en lactation; de même Waghorn et al cités par Waghorn et Hegarty (2011) n'ont pas trouvé de différence de production de méthane par $\mathrm{kg}$ de MS ingérée sur des vaches à forte ou faible ingestion résiduelle recevant des fourrages. Il y a donc un fort besoin de recherche dans ce domaine, en particulier sur vaches laitières car la plus grande partie des essais a porté sur des bovins en croissance. Dans l'état actuel des connaissances, on peut dire qu'une sélection sur l'efficacité productive réduirait probablement les émissions de méthane par $\mathrm{kg}$ de gain de poids pour les bovins en croissance ; il est toutefois nécessaire de vérifier que le classement des animaux selon leur efficacité productive est le même quelle que soit la ration distribuée.

\section{Conclusion}

Les principales pistes évoquées dans ce texte pour réduire la production de méthane sont encourageantes à des degrés divers. Pour une même proposition (addition de lipides, d'un additif spécifique) la réponse est toutefois très variable et difficile à prévoir. Les pro- grès viendront d'une meilleure compréhension des mécanismes, et donc du rôle des différents microorganismes du rumen impliqués dans la production et l'utilisation de l'hydrogène. Il est probable qu'une seule piste n'apportera pas de solution radicale. Il est donc nécessaire de continuer à explorer plusieurs pistes, pour dégager de possibles synergies entre stratégies.

Les additifs et les biotechnologies peuvent être envisagés en production intensive. Toutefois se pose le problème de leur coût, de leur acceptabilité et pour certains d'entre eux de leur difficulté d'administration, en particulier lorsque les animaux sont au pâturage. Actuellement, il n'y a pas de solution dont l'efficacité soit forte et certaine. Certaines voies sont prometteuses, soit qu'elles aient dans certains cas montré leur efficacité (plantes riches en tannins), soit qu'elles laissent supposer un potentiel de réduction de la méthanogenèse important (microorganismes exogènes). Mais des recherches supplémentaires sont indispensables, incluant des essais à long terme. Actuellement, des moyens importants sont mis sur cette thématique par des sociétés privées. En outre, il est indispensable de considérer en parallèle l'effet possible sur l'ingestion, la digestibilité de la ration et les performances: dans les conditions du terrain, l'objectif est de réduire les émissions de méthane par exploitation ou par $\mathrm{kg}$ de produit. La diminution des émissions de méthane représente un grand enjeu pour les productions extensives et en conditions tropicales et subtropicales, avec des contraintes spécifiques, comme la nécessité d'améliorer simultanément l'efficacité des productions. Les recherches sur l'effet de la nature des fourrages et l'influence de leurs métabolites secondaires sur la production de méthane sont à développer. Enfin, il est important de prendre en compte l'effet de ces stratégies sur l'émission des autres GES ou sur le stockage de carbone par les sols, et une solution pour réduire le méthane ne doit donc pas entraîner une dégradation du bilan des GES, ou d'autres impacts sur l'environnement: cette réflexion est particulièrement importante pour la mise en œuvre de stratégies visant à augmenter le pourcentage de concentré dans la ration ou la productivité des animaux. 


\section{Références}

Archimède H., Eugène M., Marie Magdeleine C., Boval M., Martin C., Morgavi D.P., Lecomte P., Doreau M., 2011. Comparison of methane production between $\mathrm{C} 3$ and C4 grasses and legumes. Anim. Feed Sci. Technol., 166-167, 59-64.

Avila J.S., Chaves A.V., Hernandez-Calva M., Beauchemin K.A., McGinn S.M., Wang Y., Harstad O.M., McAllister T.A., 2011. Effects of replacing barley grain in feedlot diets with increasing levels of glycerol on in vitro fermentation and methane production. Anim. Feed Sci. Technol., 166-167, 265-268.

Beauchemin K.A., McGinn S.M., 2005. Methane emissions from feedlot cattle fed barley or corn diets. J. Anim. Sci., 83, 653-661.

Beauchemin K.A., McGinn S.M., 2006. Methane emissions from beef cattle: effects of fumaric acid, essential oil, and canola oil. J. Anim. Sci., 84, 1489-1496.

Beauchemin K.A., McGinn S.M., Martinez T.F., McAllister T.A., 2007. Use of condensed tannin extract from quebracho trees to reduce methane emissions from cattle. J. Anim. Sci. 85, 1990-1996.

Beauchemin K.A., Kreuzer M., O’Mara F., McAllister T.A., 2008. Nutritional management for enteric methane abatement: a review. Austr. J. Exp. Agric., 48, 21-27.

Beauchemin K.A., McAllister T.A., McGinn, S.M., 2009. Dietary mitigation of enteric methane from cattle. CAB Reviews: Persp. Agric. Vet. Sci. Nutr. Nat. Res. 4, CABI, Wallingford, Royaume-Uni, 4, 1-18.

Beever D.E., Thomson D.J., Ulyatt M.J., Cammell S.B., Spooner M.C., 1985. The digestion of fresh perennial ryegrass (Lolium perenne L. cv. Melle) and white clover (Trifolium repens L. cv. Blanca) by growing cattle fed indoors. Brit. J. Nutr., 54, 763-775.

Beever D.E., Cammell S.B., Sutton J.D., Spooner M.C., Haines M.J, Harland J.I. 1989. Effects of concentrate type on energy utilization in lactating dairy cows. In: Energy metabolism of farm animals. Van der Honing Y., Close W.H. (Eds). Pudoc, Wageningen, Pays-Bas, EAAP Publ. 43, 33-36.

Benchaar C., Greathead H., 2011. Essential oils and opportunities to mitigate enteric methane emissions from ruminants. Anim. Feed Sci. Technol., 166-167, 338-355.

Blaxter K.L., Clapperton J.L., 1965. Prediction of the amount of methane produced by ruminants. Brit. J. Nutr., 19, 511-522.

Bodas R., Lopez S., Fernandez M., GarciaGonzalez R., Rodriguez A.B., Wallace R.J., Gonzalez J.S., 2008. In vitro screening of the potential of numerous plant species as antimethanogenic feed additives for ruminants. Anim. Feed Sci. Technol., 145, 245-258.

Brown E.G., Anderson R.C., Carstens G.E., Guttierez-Banuelos H., McReynolds J.L., Slay L.J., Callaway T.R., Nisbet D.J., 2011. Effects of oral nitroethane administration on enteric methane emissions and ruminal fermentation in cattle. Anim. Feed Sci. Technol., 166-167, 275-281.

Carulla J.E., Kreuzer M., Machmüller A., Hess H.D., 2005. Supplementation of Acacia mearnsii tannins decreases methanogenesis and urinary nitrogen in forage-fed sheep. Austr. J. Agric. Res., 56, 961-970.

Chaucheyras-Durand F., Walker N.D., Bach A., 2008. Effects of active dry yeasts on the rumen microbial ecosystem: Past, present and future. Anim. Feed Sci. Technol., 145, 5-26.

Chung Y.H., He M.L., McGinn S.M., McAllister T.A., Beauchemin K.A., 2011a. Linseed suppresses enteric methane emissions from cattle fed barley silage, but not from those fed grass hay. Anim. Feed Sci. Technol., 166167, 321-329.

Chung Y.H., Walker N.D., McGinn S.M., Beauchemin K.A., 2011b. Differing effects of 2 active dried yeast (Saccharomyces cerevisiae) strains on ruminal acidosis and methane production in nonlactating dairy cows. J. Dairy Sci., 94, 2431-2439.

Cottle D.J., Nolan J.V., Wiedemann S.G., 2011. Ruminant enteric methane mitigation: a review. Anim. Prod. Sci., 51, 491-514.

Dollé J.B., Agabriel J., Peyraud J.L., Faverdin P., Manneville V., Raison C., Gac A., Le Gall A., 2011. Les gaz à effet de serre en élevage bovin : évaluation et leviers d'action. In : Gaz à effet de serre en élevage bovin : le méthane. Doreau M., Baumont R., Perez J.M. (Eds). Dossier, INRA Prod. Anim. 24, 415-432.

Doreau M., Dollé J.B., 2011. Strategies for reducing greenhouse gas emissions in dairy production. A European perspective. Proc. Eastern Nutr. Conf., Montréal, Canada, 12-13 avril, 20 p.

Doreau M., Jouany J.P., 1998. Effect of a Saccharomyces cerevisiae culture on nutrient digestion in lactating dairy cows. J. Dairy Sci., 81, 3214-3221.

Doreau M., Bauchart D., Chilliard Y., 2011a. Enhancing fatty acid composition of milk and meat through animal feeding. Anim. Prod. Sci. $51,19-29$

Doreau M., van der Werf H.M.G., Micol D., Dubroeucq H., Agabriel J., Rochette Y., Martin C., 2011b. Enteric methane production and greenhouse gases balance of diets differing in concentrate in the fattening phase of a beef production system. J. Anim. Sci., 89, 2518-2528.

Eugène M., Benchaar C., Chiquette J., Massé D., 2008. Meta-analysis on the effects of lipid supplementation on methane production of lactating dairy cows. Can. J. Anim. Sci. 88, 331-337.

Eugène M., Martin C., Mialon M.M., Krauss D., Renand G., Doreau M., 2011. Dietary linseed and starch supplementation decreases methane production of fattening bulls. Anim. Feed Sci. Technol., 166-167, 330-337.

Fievez V., Boeckaert C., Vlaeminck B., Mestdagh J., Demeyer D., 2007. In vitro examination of DHA-edible micro-algae: 2 . Effect on rumen methane production and apparent degradability of hay. Anim. Feed Sci. Technol., $136,80-95$

Garcia-Gonzalez R., Lopez S., Fernandez M., Bodas R., Gonzalez J.S., 2008. Screening the activity of plants and spices for decreasing ruminal methane production in vitro. Anim. Feed Sci. Technol., 147, 36-52.

Garnsworthy P.C., 2004. The environmental impact of fertility in dairy cows: a modelling approach to predict methane and ammonia emissions. Anim. Feed Sci. Technol., 112, 211 223.

Gerber P., Vellinga T., Opio C., Steinfeld H., 2011. Productivity gains and greenhouse gas emissions intensity in dairy systems. Livest. Sci., 139, 100-108.

Giger-Reverdin S., Morand-Fehr P., Tran G., 2003. Literature survey of the influence of dietary fat composition on methane production in dairy cattle. Livest. Prod. Sci., 82, 73-79.

Grainger C., Clarke T., Auldist M.J., Beauchemin K.A., McGinn S.M., Waghorn G.C., Eckard R.J., 2009. Potential use of Acacia mearnsii condensed tannins to reduce methane emissions and nitrogen excretion from grazing dairy cows. Can. J. Anim. Sci., $89,241-251$

Grainger C., Williams R., Clarke T., Wright A.D.G., Eckard R.J., 2010. Supplementation with whole cottonseed causes long-term reduction of methane emissions from lactating dairy cows offered a forage and cereal grain diet. J. Dairy Sci., 93, 2612-2619.

Grainger C., Beauchemin K.A., 2011. Can enteric methane emissions from ruminants be lowered without lowering their production? Anim. Feed Sci. Technol., 166-167, 308-320.

Guan H., Wittenberg K.M., Ominski K.H., Krause D.O., 2006. Efficacy of ionophores in cattle diets for mitigation of enteric methane. J. Anim. Sci., 84, 1896-1906.

Hammond K.J., Hoskin S.O., Burke J.L., Waghorn G.C., Koolaard J.P., Muetzel S., 2011. Effects of feeding fresh white clover (Trifolium repens) or perennial ryegrass (Lolium perenne) on enteric methane emissions from sheep. Anim. Feed Sci. Technol., 166-167, 398-404.

Hegarty R.S., Goopy J.P., Herd R.M., McCorkell B., 2007. Cattle selected for lower residual feed intake have reduced daily methane production. J. Anim. Sci., 85, 14791486.

Hess H.D., Beuret R.A., Lötscher M., Hindrichsen I.K., Machmüller A., Carulla J.E., Lascano C.E., Kreuzer M., 2004. Ruminal fermentation, methanogenesis and nitrogen utilization of sheep receiving tropical grass hayconcentrate diets offered with Sapindus saponaria fruits and Cratylia argentea foliage. Anim. Sci., 79, 177-189.

Holter J.B., Hayes H.H., Urban W.E. Jr., Duthie A.H., 1992. Energy balance and lactation response in Holstein cows supplemented with cottonseed with or without calcium soap. J. Dairy Sci., 75, 1480-1494.

Holtshausen L., Chaves A.V., Beauchemin K.A., McGinn S.M., McAllister T.A., Odongo N.E., Cheeke P.R., Benchaar C., 2009. Feeding saponin-containing Yucca schidigera and Quillaja saponaria to decrease enteric methane production in dairy cows. J. Dairy Sci., 92, 2809-2821.

IPCC., 2006. Emissions from livestock and manure management. IPCC Guidelines for National Greenhouse Inventories, chapter 10: http://www.ipcc-nggip.iges.or.jp/public/ $\underline{2006 \mathrm{gl} /}$

Jayanegara A., Leiber F., Kreuzer M., 2011a. Meta-analysis of the relationship between dietary tannin level and methane formation in 
ruminants from in vivo and in vitro experiments. J. Anim. Physiol. Anim. Nutr., paru en ligne, doi: 10.1111/j.1439-0396.2011.01172.x

Jayanegara A., Wina E., Soliva C.R., Marquardt S., Kreuzer M., Leiber F., 2011b. Dependence of forage quality and methanogenic potential of tropical plants on their phenolic fractions as determined by principal component analysis. Anim. Feed Sci. Technol., 163, 231-243.

Joblin K.N., 1999. Ruminal acetogens and their potential to lower ruminant methane emissions. Austr. J. Agric. Res., 50, 1307-1313.

Johnson K.A., Johnson D.E., 1995. Methane emissions from cattle. J. Anim. Sci., 73, 24832492.

Jones F.M., Phillips F.A., Naylor T., Mercer N.B., 2011. Methane emissions from grazing Angus beef cows selected for divergent residual feed intake. Anim. Feed Sci. Technol., 166-167, 302-307.

Jouany J.P., 1994. Les fermentations dans le rumen et leur optimisation. INRA Prod. Anim., 7, 207-225.

Jouany J.P., Papon Y., Morgavi D.P., Doreau M., 2008. Linseed oil and a combination of sunflower oil and malic acid decrease rumen methane emissions in vitro. In: Livestock and global climate change. Rowlinson P., Steele M., Nefzaoui A. (Eds). Cambridge University Press, Cambridge, Royaume-Uni, 140-143.

Kirkpatrick D.E. Steen R.W.J. 1999. The effect of method of conservation of grass and supplementation on energy and nitrogen utilization by lambs. Anim. Sci., 133, 409-417.

Klevenhusen F., Zeitz J.O., Duval S. Kreuzer M., Soliva C.R., 2011. Garlic oil and its principal component diallyl disulfide fail to mitigate methane, but improve digestibility in sheep. Anim. Feed Sci. Technol., 166-167, 356-363.

Klieve A., Joblin K., 2007. Comparison in hydrogen utilisation of ruminal and marsupial reductive acetogens. In: The Pastoral Greenhouse Gas Research Consortium. Kennedy R. (Ed). Wellington, NouvelleZélande, 34-35.

Lovett D.K., Lovell S., Stack L., Callan J., Finlay M., Conolly J., O'Mara F.P., 2003. Effect of forage/concentrate ratio and dietary coconut oil level on methane output and performance of finishing beef heifers. Livest. Prod. Sci., 84, 135-146.

Macheboeuf D., Morgavi D.P., Papon Y., Mousset J.L., Arturo-Schaan M., 2008. Doseresponse effects of essential oils on in vitro fermentation activity of the rumen microbial population. Anim. Feed Sci. Technol., 145, 335-350.

Machmüller A., Soliva C.R., Kreuzer M., 2003. Methane-suppressing effect of myristic acid in sheep as affected by dietary calcium and forage proportion. Brit. J. Nutr., 90, 529-540.

Mao H.L., Wang J.K., Zhou Y.Y., Liu J.X., 2010. Effects of addition of tea saponins and soybean oil on methane production, fermentation and microbial population in the rumen of growing lambs. Livest. Sci., 129, 56-62.

Martin C., Rouel J., Jouany J.P., Doreau M., Chilliard Y., 2008. Methane output from dairy cows in response to dietary supplementation of crude linseed, extruded linseed or linseed oil. J. Anim. Sci., 86, 2642-2650.
Martin C., Ferlay A., Chilliard Y., Doreau M., 2009. Decrease in methane emissions in dairy cows with dietary linseed content. Proc. Ann. Meet. Brit. Soc. Anim. Sci., Southport, Royaume-Uni, 21.

Martin C., Morgavi D.P., Doreau M., 2010. Methane mitigation in ruminants: from microbe to the farm scale. Animal, 4, 351-365.

Martin C., Pomiès D., Ferlay A., Rochette Y., Martin B., Chilliard Y., Morgavi D.P., Doreau M., 2011. Methane output and rumen microbiota in dairy cows in response to longterm supplementation with linseed or rapeseed of grass silage- or pasture-based diets. Proc. N. Z. Soc. Anim. Prod., 71, 242-247.

McCaughey W.P., Wittenberg K., Corrigan D., 1999. Impact of pasture type on methane production by lactating beef cows. Can. J. Anim. Sci., 79, 221-226.

McCourt A.R., Yan T., Mayne S., Wallace J., 2008. Effect of dietary inclusion of encapsulated fumaric acid on methane production from grazing dairy cows. Proc. Ann. Meet. Brit. Soc. Anim. Sci., Scarborough, Royaume-Uni, 64.

McGinn S.M., Chung Y.H., Beauchemin K.A., Iwaasa A.D., Grainger C., 2009. Use of corn distillers' dried grains to reduce enteric methane loss from beef cattle. Can. J. Anim. Sci., 89, 409-413.

Moate P.J., Williams S.R.O., Grainger C. Hannah M.C., Ponnampalam E.N., Eckard R.J., 2011. Influence of cold-pressed canola, brewers grains and hominy meal as dietary supplements suitable for reducing enteric methane emissions from lactating dairy cows. Anim. Feed Sci. Technol., 166-167, 254-264.

Moe P.W., Tyrrell H.F., 1979. Methane Production in Dairy Cows. J. Dairy Sci., 62, 1583-1586.

Moe P.W., Tyrrell H.F., Hooven N.W. Jr., 1973. Energy balance measurements with corn meal and ground oats for lactating cows. J. Dairy Sci., 56, 1149-1153.

Mohammed N., Ajisaka N., Lila Z.A., Koji Hara, Mikuni, K., Hara K., Kanda S., Itabashi H., 2004. Effect of Japanese horseradish oil on methane production and ruminal fermentation in vitro and in steers. J. Anim. Sci., 82, 18391846.

Moran D., MacLeod M., Wall E., Eory V., McVittie A., Barnes A., Rees R.M., Topp C.F.E., Pajot G., Matthews R., Smith P., Moxey A., 2011. Developing carbon budgets for UK agriculture, land-use, land-use change and forestry out to 2022. Climatic Change, $105,529-553$.

Morgavi D.P., Forano E., Martin C., Newbold C.J., 2010a. Microbial ecosystem and methanogenesis in ruminants. Animal, 4, 1024 1036.

Morgavi D.P., Martin C., Boudra H., 2010 b. Fungal secondary metabolites reduce rumen methane production in vitro and in vivo. McGeough E.J., McGinn S.M. (Eds). Banff, Canada, Proc. Greenhouse Gases Anim. Agric., 162.

Münger A., Kreuzer M., 2008. Absence of persistent methane emission differences in three breeds of dairy cows. Austr. J. Exp. Agric., 48, 77-82.

Newbold C.J., Lopez S., Nelson N., Ouda J.O., Wallace R.J., Moss A.R., 2005. Propionate precursors and other metabolic intermediates as possible alternative electron acceptors to methanogenesis in ruminal fermentation in vitro. Brit. J. Nutr., 94, 27-35.

Newbold C.J., Rode L.M., 2006. Dietary additives to control methanogenesis in the rumen. Soliva C.R., Takahashi J., Kreuzer M. (Eds). Elsevier Int. Congr. Series 1293, Elsevier, Amsterdam, Pays-Bas, Proc. Greenhouse Gases Anim. Agric., 138-147.

Niderkorn V., Martin C., Le Morvan A., Rochette Y., Brison A., Baumont R., 2011. Effect of increasing proportion of alfalfa in alfalfa-ryegrass mixed diets on intake, digestion and methane emissions in sheep. Proc. $8^{\text {th }}$ Int. Symp. Nut. Herb., 6-9 septembre, Aberystwyth, Royaume-Uni, 563.

Nkrumah J.D., Okine E.K., Mathison J.W., Schmid K., Li C., Basarab J.A., Price M.A., Wang Z., Moore S.S., 2006. Relationships of feedlot feed efficiency, performance, and feeding behavior with metabolic rate, methane production, and energy partitioning in beef cattle. J. Anim. Sci., 84, 145-153.

Odongo N.E., Bagg R., Vessie G., Dick P. Or-Rashid M.M., Hook S.E., Gray J.T., Kebreab F., France J., McBride B.W., 2007. Long-term effects of feeding monensin on methane production in lactating dairy cows. $\mathrm{J}$. Dairy Sci., 90, 1781-1788.

Patra A.K., Kamra D.N., Bhar R., Kumar P., Agarwal N., 2011. Effect of Terminalia chebula and Allium sativum on in vivo methane emission by sheep. J. Anim. Physiol. Anim. Nutr., 95, 187-191.

Patra A.K., Saxena J., 2010. A new perspective on the use of plant secondary metabolites to inhibit methanogenesis in the rumen. Phytochemistry, 71, 1198-1222.

Paul S.S., Deb S.M., Singh D., 2011. Isolation and characterization of novel sulphate-reducing Fusobacterium sp. and their effects on in vitro methane emission and digestion of wheat straw by rumen fluid from Indian riverine buffaloes. Anim. Feed Sci. Technol., $166-167,132-140$

Pen B., Takaura K., Yamaguchi S., Asa R., Takahashi J., 2007. Effects of Yucca schidigera and Quillaja saponaria with or without $\beta$ 1-4 galacto-oligosaccharides on ruminal fermentation, methane production and nitrogen utilization in sheep. Anim. Feed Sci. Technol., 138, 75-88.

Pinarès-Patiño C.S., Baumont R., Martin C., 2003. Methane emissions by charolais cows grazing a monospecific pasture of timothy at four stages of maturity. Can. J. Anim. Sci., 83, 769-777.

Pinares-Patiño C.S. Waghorn G.C., Machmuller A., Vlaming B., Molano G., Cavanagh A., Clark H., 2007. Methane emissions and digestive physiology of non-lactating dairy cows fed pasture forage. Can. J. Anim. Sci., 87, 601-613.

Pinares-Patiño C.S., McEwan J.C., Dodds K.G., Cárdenas E.A., Hegarty R.S., Koolaard J.P., Clark H., 2011. Repeatability of methane emissions from sheep. Anim. Feed Sci. Technol., 166-167, 210-218.

Popova M., Morgavi D.P., Doreau M., Martin C., 2011. Production de méthane et interactions microbiennes dans le rumen. In : Gaz à effet de serre en élevage bovin : le méthane. Doreau M., Baumont R, Perez J.M. (Eds). Dossier, INRA Prod. Anim. 24, 447-460.

Robertson L.J., Waghorn G.C., 2002. Dairy industry perspectives on methane emissions and production from cattle fed pasture or total 
mixed rations in New Zealand. Proc. N.Z. Soc. Anim. Prod., 62, 213-218.

Sauvant D., Giger-Reverdin S., 2007. Empirical modelling meta-analysis of digestive interactions and $\mathrm{CH}_{4}$ production in ruminants. In: Energy and protein metabolism and nutrition, Wageningen Academic Publishers, Wageningen, Pays-Bas, EAAP Publ., 124, 561.

Sauvant D., Giger-Reverdin S., 2009. Modélisation des interactions digestives et de la production de méthane chez les ruminants. INRA Prod. Anim., 22, 375-384.

Sauvant D., Giger-Reverdin S., Serment A., Broudiscou L., 2011. Influences des régimes et de leur fermentation dans le rumen sur la production de méthane par les ruminants. In : Gaz à effet de serre en élevage bovin : le méthane. Doreau M., Baumont R., Perez J.M. (Eds). Dossier, INRA Prod. Anim. 24, 433-446.

Shinkai T., Mitsumori M., Enishi O., Takenaka A., Kobayashi, Y., 2010. Monitoring of methane and hydrogen production from the rumen of cows fed cashew (Anacardium occidentale) nut shell liquid. McGeough E.J., McGinn S.M. (Eds). Banff, Canada, 3-8 octobre, Proc. Greenhouse Gases Anim. Agric., 152.

Sliwinski B.J., Kreuzer M., Wettstein H.R., Machmüller A., 2002. Rumen fermentation and nitrogen balance of lambs fed diets containing plant extracts rich in tannins and saponins, and associated emissions of nitrogen and methane. Arch. Anim. Nutr., 56, 379-392.

Soliva C.R., Zeleke A.B., Clément C., Hess H.D., Fievez V., Kreuzer M., 2008. In vitro screening of various tropical foliages, seeds, fruits and medicinal plants for low methane and high ammonia generating potentials in the rumen. Anim. Feed Sci. Technol., 147, 53-71.

Staerfl S.M., Zeitz J.O., Kreuzer M., Soliva C.R., 2012. Methane conversion rate of bulls fattened on grass or maize silage as compared with the IPCC default values, and the longterm methane mitigation efficiency of adding acacia tannin, garlic, maca and lupine . Agric. Ecosyst. Environ., 148, 111-120.

Tekippe J.A., Hristov A.N., Heyler K.S., Cassidy T.W., Zheljazkov V.D., Ferreira J.F.S., Karnati S.K., Varga, G.A., 2011. Rumen fermentation and production effects of Origanum vulgare $L$. leaves in lactating dairy cows. J. Dairy Sci., 94, 5065-5079.
Ungerfeld E.M., Forster R.J., 2011. A metaanalysis of malate effects on methanogenesis in ruminal batch cultures. Anim Feed Sci. Technol., 166-167, 282-290.

Van Dorland H.A., Wettstein H.R., Leuenberger H., Kreuzer M., 2007. Effect of supplementation of fresh and ensiled clovers to ryegrass on nitrogen loss and methane emissions in dairy cows. Livest. Sci., 111, 57-69.

Van Kessel J.A.S., Russell J.B., 1996. The effect of $\mathrm{pH}$ on ruminal methanogenesis. FEMS Microbiol. Ecol., 20, 205-210.

Van Zijderveld S.M., Gerrits W.J.J., Apajalahti J.A., Newbold J.R., Dijkstra J., Leng R.A. Perdok H.B., 2010a. Nitrate and sulfate: Effective alternative hydrogen sinks for mitigation of ruminal methane production in sheep. J. Dairy Sci., 93, 5856-5866.

Van Zijderveld S.M., Dijkstra J., Gerrits W.J.J., Newbold J.R., Perdok H.B., 2010b. Dietary nitrate persistently reduces enteric methane production in lactating dairy cows. McGeough E.J., McGinn S.M. (Eds). 3-8 octobre, Banff, Canada, Proc. Greenhouse Gases Anim. Agric., 157.

Van Zijderveld S.M., Fonken B., Dijkstra J., Gerrits W.J.J., Perdok H.B., Fokking W., Newbold J.R., 2011. Effects of a combination of feed additives on methane production, diet digestibility, and animal performance in lactating dairy cows. J. Dairy Sci., 94, 1445-1454.

Vermorel M., 1995. Emissions annuelles de méthane d'origine digestive par les bovins en France. Variations selon le type d'animal et le niveau de production. INRA Prod. Anim., 8, 265-272.

Vermorel M., Jouany J.P., Eugène M. Sauvant D., Noblet J., Dourmad J.Y., 2008. Evaluation quantitative des émissions de méthane entérique par les animaux d'élevage en 2007 en France. INRA Prod. Anim., 21 403-418.

Vlaming J.B., Lopez-Villalobos N., Brookes I.M., Hoskin S.O., Clark H., 2008. Within- and between-animal variance in methane emissions in non-lactating dairy cows. Austr. J. Exp. Agric., 48, 124-127.

Waghorn G., 2008. Beneficial and detrimental effects of dietary condensed tannins for sustainable sheep and goat production - Progress and challenges. Anim. Feed Sci. Technol., 147, 116-139.

Waghorn G.C., Hegarty R.S., 2011. Lowering ruminant methane emissions through improved feed conversion efficiency. Anim. Feed Sci. Technol., 166-167, 291-301.

Wallace R.J., Wood T.A., Rowe A., Price J., Yanez D.R., Williams S.P., Newbold C.J., 2006. Encapsulated fumaric acid as a means of decreasing ruminal methane emissions. an update. Soliva C.R., Takahashi J., Kreuzer M. (Eds). Elsevier Int. Congr. Series 1293 Elsevier Amsterdam, Pays-Bas, Proc. Greenhouse Gases Anim. Agric. an update, 148-151.

Wang C.J., Wang S.P., Zhou H., 2009. Influences of flavomycin, ropadiar, and saponin on nutrient digestibility, rumen fermentation, and methane emission from sheep. Anim. Feed Sci. Technol., 148, 157-166.

Woodward S.L., Waghorn G.C., Lassey K. R., Laboyrie P.G., 2002. Does feeding sulla (Hedysarum coronarium) reduce methane emissions from dairy cows? Proc. N.Z. Soc. Anim. Prod., 62, 227-230.

Woodward S.L., Waghorn G.C., Laboyrie P.G., 2004. Condensed tannins in birdsfoot trefoil (Lotus corniculatus) reduce methane emissions from dairy cows. Proc. N.Z. Soc. Anim. Prod., 64, 160-164.

Woodward S.L., Waghorn G.C., Thomson N.A., 2006. Supplementing dairy cows with oils to improve performances and reduce methane - does it work? Proc. N.Z. Soc. Anim. Prod., 66, 176-181.

Zhou M., Hernandez-Sanabria E., Guan L.L., 2010. Characterization of variation in rumen methanogenic communities under different dietary and host feed efficiency conditions, as determined by PCR-Denaturing Gradient Gel Electrophoresis analysis. Appl. Environ. Microbiol., 76, 3776-3786.

Zhou Y.Y., Mao H.L., Jiang F., Wang J.K., Liu J.X., McSweeney C.S., 2011. Inhibition of rumen methanogenesis by tea saponins with reference to fermentation pattern and microbial communities in $\mathrm{Hu}$ sheep. Anim. Feed Sci. Technol., 166-167, 93-100.

\section{Résumé}

Cet article fait le point des connaissances sur différentes pistes pour réduire la production de méthane entérique par les ruminants, en privilégiant les approches in vivo, et évalue les possibilités d'application pratique. Il est connu que l'accroissement du pourcentage de concentré dans la ration réduit la production de méthane par $\mathrm{kg}$ de matière sèche ingérée. Cet effet est toutefois plus sensible pour des proportions élevées de concentré. Les céréales diminuent plus la production de méthane que les concentrés riches en fibres. L'addition de lipides à la ration a un effet significatif pour réduire les émissions de méthane. Parmi les fourrages, l'ensilage de maïs entraîne des émissions plus faibles que l'ensilage d'herbe ou le foin ; mais les différences induites entre stades de végétation de l'herbe ou entre graminées et légumineuses sont faibles, et parfois controversées. De très nombreux extraits de plantes et autres additifs aux modes d'action divers ont été testés. Les plantes riches en tannins et les tannins extraits des plantes sont efficaces pour réduire le méthane mais le risque de diminution de la digestibilité de la ration doit être considéré. Les autres additifs nécessitent des études supplémentaires pour attester leur efficacité sur le long terme. Parmi les biotechnologies, celles visant à réorienter la production d'hydrogène vers d'autres produits que le méthane sont séduisantes, mais pas encore mises au point. L'accroissement du niveau de production des animaux entraîne une réduction de la production de méthane qui est particulièrement marquée quand elle est rapportée au kg de lait ou de viande. Enfin, il y a des différences entre animaux dans l'efficacité globale de production, appréciée par l'ingestion rapportée à un même niveau de production. Les animaux les plus efficaces produisent moins de méthane par kg de lait ou de viande. L'article met l'accent sur la nécessité de ne pas considérer la diminution du méthane seule, mais d'évaluer l'efficacité des stratégies proposées sur l'ensemble des GES. 


\begin{abstract}
Tools for decreasing enteric methane production by ruminants

This paper reviews the strategies to reduce methanogenesis in ruminants and their applicability on farm. It is known that increasing the percentage of concentrate decreases the production of methane per $\mathrm{kg}$ of dry matter intake. This effect is especially important at high levels of concentrate. Cereals decrease more methane emission than concentrates rich in fibre. Lipid inclusion in diets significantly decreases methane emission. Among forages, maize silage diets result in lower emissions than hay or grass silage diets, but differences in vegetation stages or between grasses and legumes are low and sometimes controversial. Alarge number of plant extracts and additives with different modes of action have been assayed. Plants rich in tannins or tannin extracts result in methane mitigation, but the risk of impairing diet digestibility has to be considered. The long term efficacy of many of these additives still needs to be evaluated. Biotechnologies that reduce dihydrogen available for methanogenesis are promising but need further testing. Increasing the level of production of animals results in a decrease in methane production, which is especially significant when methane is expressed per kg of milk or meat. There are also differences between animals in their global productive efficiency, evaluated through the residual feed intake. The most efficient animals produce less methane per $\mathrm{kg}$ of milk or meat. This paper stresses the need to look beyond enteric methane mitigation, and to evaluate the efficacy of mitigation strategies on all greenhouse gases associated to ruminant production.
\end{abstract}

DOREAU M., MARTIN C., EUGÈNE M., POPOVA M., MORGAVI D.P., 2011. Leviers d'action pour réduire la production de méthane entérique par les ruminants. In : Gaz à effet de serre en élevage bovin : le méthane. Doreau M., Baumont R., Perez J.M. (Eds). Dossier, INRA Prod. Anim. 24, 461-474. 